\title{
Microanalyse nucléaire.
}

\author{
François Abel, \\ GPS, Universités Paris 6 et Paris 7, UMR 7588 du CNRS, \\ Tour 23, 2 place Jussieu, F-75251 PARIS CEDEX 05, FRANCE \\ abel@gps.jussieu.fr
}

\section{INTRODUCTION}

La microanalyse nucléaire est un ensemble de techniques qui permettent l'analyse élémentaire et isotopique qualitative et quantitative de la région superficielle des solides, indépendamment des liaisons chimiques. La profondeur analysée est dans la gamme du micromètre et est donc bien adaptée à l'analyse des couches minces. Toutes ces techniques utilisent des faisceaux d'ions légers monocinétiques $\left({ }^{1} \mathrm{H},{ }^{2} \mathrm{H},{ }^{3} \mathrm{He},{ }^{4} \mathrm{He}\right.$ dans la grande majorité des cas) d'une énergie de l'ordre du $\mathrm{MeV}$, c'est à dire entre $100 \mathrm{keV}$ et quelques $\mathrm{MeV}$.

Les informations sur la composition sont extraites du nombre, de la nature et du spectre en énergie des particules (ions ou photons $\mathrm{X}$ ou $\gamma$ ) émises et détectées pendant l'irradiation du matériau par le faisceau d'analyse, dans des conditions expérimentales bien définies par l'énergie du faisceau, le nombre d'ions incidents et la géométrie et les conditions d'irradiation et de détection. L'échantillon analysé par ces techniques ne subit que peu ou pas de dégâts.

Le champ d'application de ces techniques comprend la physique des matériaux, la physicochimie et la chimie, la géophysique, l'archéologie, les études d'œuvres d'art ainsi que les études d'environnement.

\section{INTERACTIONS IONS-MATIÈRE}

\subsection{Généralités}

Ces techniques reposent sur l'utilisation des phénomènes se produisant lors du passage des ions dans la matière. Globalement, les ions perdent leur énergie par transfert au milieu traversé et finissent, si l'épaisseur de la cible est suffisante, par rester implantés. Les transferts d'énergie engendrent dans le milieu différents types de phénomènes : excitations électroniques et ionisations, mises en mouvement des atomes. Les déplacements atomiques primaires, créés à la suite de collisions ion-atome avec un transfert d'énergie suffisant, peuvent engendrer des cascades de collisions et de déplacements atomiques et donc des défauts. Les cascades de collisions peuvent aboutir à la pulvérisation des atomes de la surface, phénomène particulièrement important pour les ions assez lourds et de basse vitesse. En conséquence des ionisations, on observe l'émission d'électrons secondaires et de photons. Lorsque le paramètre d'impact est très petit, donc très rarement, des phénomènes particuliers se produisent : diffusion élastique à grand angle de l'ion incident, émission de rayons $\mathrm{X}$ due à une ionisation en couche interne, ou réaction nucléaire induite par la formation d'un noyau composé à partir des noyaux de l'ion incident et d'un atome du milieu et sa désintégration produisant une particule et/ou un photon $\gamma$. 


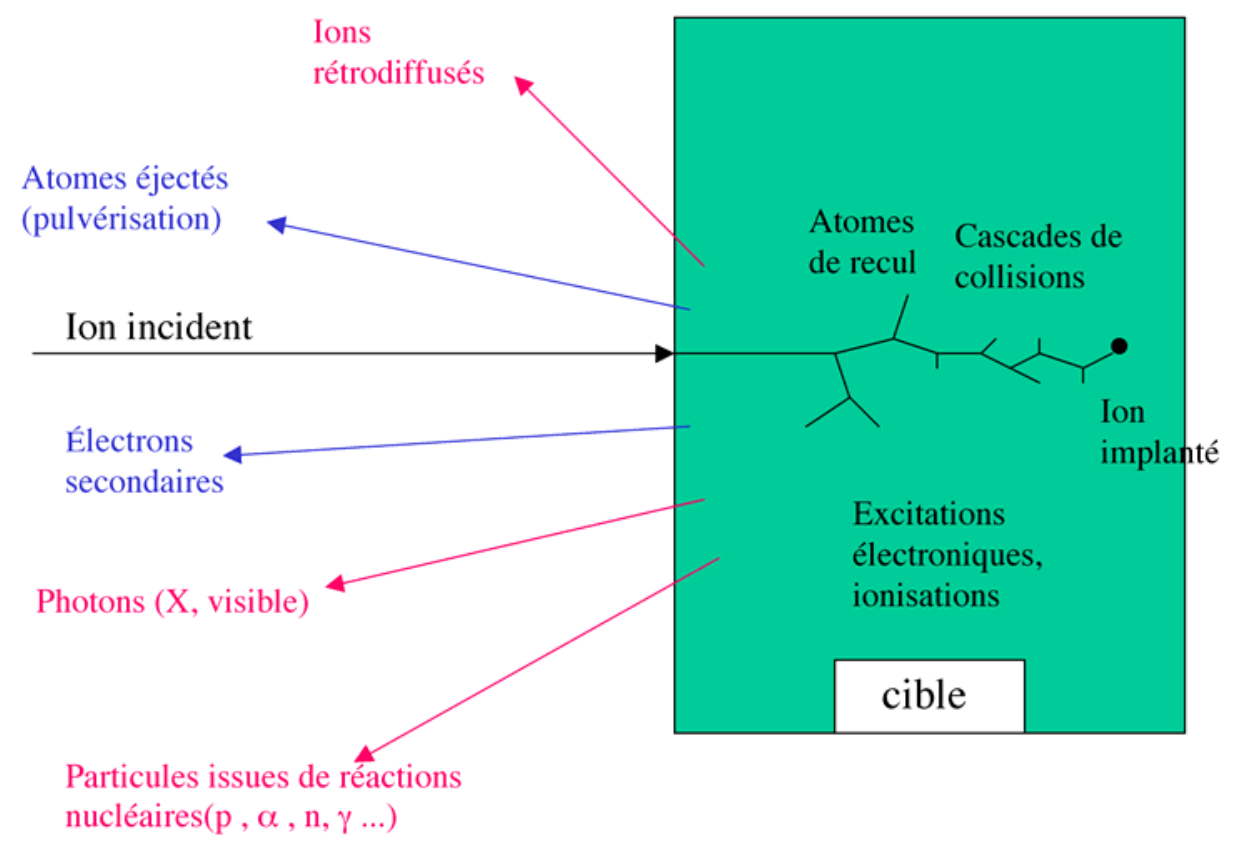

FIG. 1. Résumé schématique des événements se produisant lors du passage d'un ion à travers la matière.

\subsection{Pertes d'énergie de l'ion incident}

On peut distinguer deux mécanismes de perte d'énergie : la perte «nucléaire » où le transfert est élastique et se fait de l'ion incident à l'atome considéré comme un tout et la perte "électronique », inélastique, où le transfert correspond à des excitations ou des ionisations des électrons des atomes du milieu. La qualification de " nucléaire» dans le premier cas est justifiée par le fait que l'énergie, de type cinétique, gagnée par l'atome cible est portée essentiellement par le noyau eu égard au rapport des masses noyau-électrons.

Ces pertes d'énergie sont caractérisées par la grandeur « pouvoir d'arrêt» $\frac{\mathrm{d} E}{\mathrm{~d} x} \mathrm{du}$ milieu pour l'ion considéré. Il s'exprime en quantité d'énergie perdue par unité de quantité de matière par unité de surface traversée. Souvent exprimée en $\mathrm{MeV} \cdot \mathrm{cm}^{2} / \mathrm{g}\left(\mathrm{MeV} /\left(\mathrm{g} / \mathrm{cm}^{2}\right)\right)$, l'unité naturelle de cette grandeur est plutôt l'eV pour $10^{15}$ atomes par $\mathrm{cm}^{2}$.

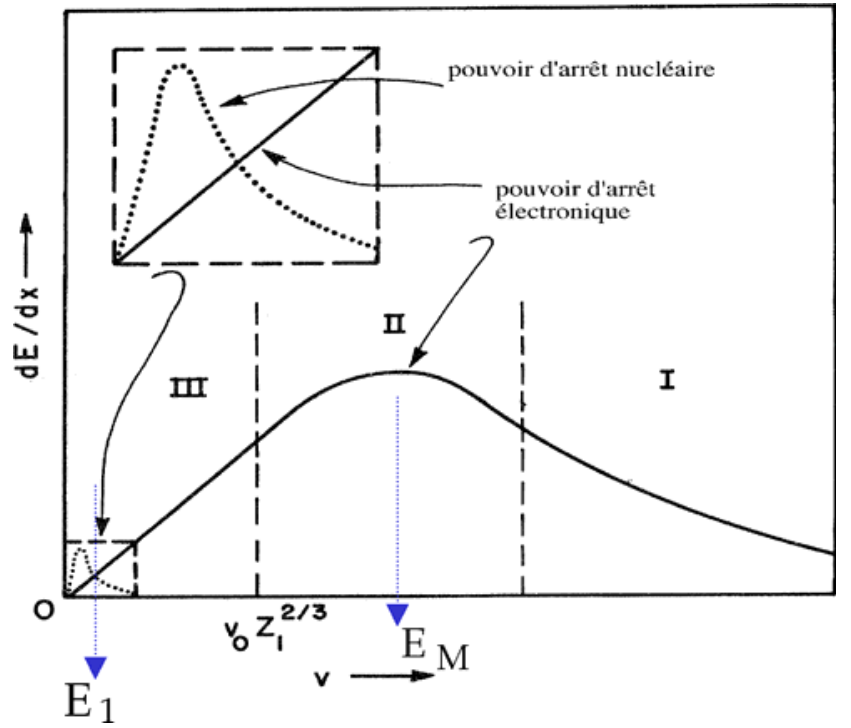

FIG. 2. Pertes d'énergie des ions dans la matière en fonction de la vitesse de l'ion. 


\subsubsection{Pertes d'énergie aux basses vitesses}

Dans le domaine des interactions ion-matière, la dénomination «basses vitesses » correspond à la gamme d'énergie où l'ion possède une vitesse inférieure à $v_{o} Z_{1}^{2 / 3}$, où $Z_{1}$ est le numéro atomique de l'ion et $v_{o}$ est la vitesse de Bohr $\frac{e^{2}}{\hbar}$ qui est celle que l'on peut associer à l'électron de l'hydrogène sur son orbite (région III de la figure 2). Dans ce cas, l'ion incident conserve sa charge initiale.

\subsubsection{Pertes d'énergie nucléaires}

Ces transferts d'énergie correspondent aux collisions élastiques ion-atome. Le potentiel d'interaction est le potentiel coulombien d'interaction entre les noyaux écranté par les électrons de l'atome et éventuellement de l'ion. L'intégration sur tous les paramètres d'impact donne la perte d'énergie représentée dans l'insert de la figure 2 en fonction de la vitesse de l'ion. Cette perte d'énergie passe par un maximum et décroît. Ce type de perte d'énergie devient très rapidement petit devant les pertes électroniques lorsque la vitesse de l'ion croît vers la valeur $v_{o} Z_{1}^{2 / 3}$. Une grandeur caractéristique intéressante est la valeur de la vitesse ou celle de l'énergie $E_{1}$ pour laquelle la perte d'énergie nucléaire, en décroissant, devient égale à la perte d'énergie électronique. Elle augmente très vite avec le numéro atomique, et donc la masse de l'ion. Dans le silicium, cette valeur est égale à $8 \mathrm{eV}$ pour $\mathrm{H}^{+}, 0,9 \mathrm{keV}$ pour ${ }^{4} \mathrm{He}^{+}, 10 \mathrm{keV}$ pour ${ }^{10} \mathrm{~B}^{+}$et $200 \mathrm{keV}$ pour $\mathrm{Ar}^{+}$. La gamme d'énergie pour laquelle les pertes d'énergie nucléaires sont prédominantes est donc très variable avec la nature de l'ion.

Si l'énergie transférée est suffisante, supérieure à une dizaine d'eV, l'atome est mis en mouvement, peut mettre en mouvement lors de collisions secondaires d'autres atomes de la cible (cascades de collisions) et crée un ou plusieurs défauts (lacunes, interstitiels...). Le nombre moyen d'atomes déplacés par ion incident peut être estimé à partir de l'expression donnée par Keachin et Pease $N=T / 2 E_{d}$ où $T$ est l'énergie moyenne transférée lors de la première collision ion-atome et $E_{d}$ l'énergie nécessaire pour déplacer un atome de son site.

\subsubsection{Pertes d'énergie électroniques}

Dans cette gamme de vitesses, l'ion est suffisamment lent pour que l'on puisse considérer que les électrons du cortège ont une vitesse associée plus grande. Le mécanisme de pertes d'énergie électroniques est alors l'échange d'électrons entre l'ion et les atomes du milieu. Ceci conduit à une proportionnalité de la perte d'énergie à la vitesse de l'ion.

\subsubsection{Pertes d'énergie pour des vitesses supérieures à $v_{o} Z_{1}^{2 / 3}$}

Pour ces vitesses, la perte d'énergie nucléaire devient négligeable. Dans la région intermédiaire, correspondant à la région II sur la figure 2 , où les vitesses sont immédiatement supérieures à $v_{o} Z_{1}{ }^{2 / 3}$, la perte d'énergie passe par un maximum pour une valeur $E_{M}$ de l'énergie qui elle aussi crôit très rapidement avec le numéro atomique de l'ion. Dans le silicium, cette valeur est $70 \mathrm{keV}$ pour $\mathrm{H}^{+}$, $450 \mathrm{keV}$ pour ${ }^{4} \mathrm{He}^{+}, 2 \mathrm{MeV}$ pour ${ }^{10} \mathrm{~B}^{+}$et $30 \mathrm{MeV}$ pour $\mathrm{Ar}^{+}$. Dans cette gamme d'énergie, il y a superposition d'échanges électroniques, d'excitations et d'ionisations, l'ion incident subissant aussi des fluctuations de charge.

Aux plus hautes énergies (région I de la figure 2) les pertes diminuent quand la vitesse augmente. L'ion est entièrement déshabillé de ses électrons. L'expression, due à Bethe et Bloch,

$$
\left.\frac{d E}{d x}\right|_{\text {él. }}=\frac{4 \pi e^{2} N Z_{2} Z_{1}^{2}}{m_{e} V_{0}^{2}} \ln \left(\frac{2 m_{e} V_{0}^{2}}{\langle I\rangle}\right),
$$

donne (en système c.g.s.) une bonne approximation de la valeur moyenne de la perte d'énergie dans ce domaine. Ici, $Z_{1}$ et $Z_{2}$ sont les numéros atomiques respectivement de l'ion et des atomes, $N$ est le 
nombre d'atomes-cible par unité de volume, $m_{e}$ la masse de l'électron, $v_{o}$ la vitesse de l'ion incident et $<I>$ est une valeur moyenne de l'énergie d'ionisation des atomes cibles. négligeables.

La loi donnée ici ne concerne pas le domaine où les corrections relativistes ne sont plus

\subsubsection{Nature statistique des pertes d'énergie}

À la traversée d'une épaisseur de matière, la perte totale d'énergie est la somme d'un grand nombre de transferts d'énergie ions-matière. Le nombre d'interactions et les paramètres d'impact étant aléatoirement répartis, chaque ion ne va pas subir la même perte d'énergie. Pour un faisceau de particules identiques et initialement monocinétiques, on aura après traversée d'une épaisseur de matière une répartition statistique de l'énergie des ions (effet de "straggling »). Lorsque la quantité de matière traversée est suffisamment grande (grand nombre d'interactions par ion), la loi statistique peut être décrite par une gaussienne dont l'écart type croît proportionnellement à la racine carrée de la masse de matière traversée.

De plus, chaque interaction conduit également à une déviation angulaire dépendant aussi du paramètre d'impact. En conséquence, on aura aussi une répartition statistique de la direction du vecteur vitesse des ions.

\subsubsection{Additivité des pouvoirs d'arrêt}

Quand la cible considérée est composée de plusieurs types d'atomes, le pouvoir d'arrêt est la somme pondérée des pouvoirs d'arrêt de chaque type d'atome. On applique alors la règle de Bragg :

$$
\left[\frac{\mathrm{d} E}{\mathrm{~d} x}\right]_{\text {total }}=\frac{\sum n_{i}\left[\frac{\mathrm{d} E}{\mathrm{~d} x}\right]_{i}}{\sum n_{i}},
$$

où les $n_{i}$ représentent la composition élémentaire du milieu. Pour les milieux moléculaires, il peut être nécessaire de faire une correction à cause des liaisons chimiques.

\section{3. Événements à petit paramètre d'impact}

Parmi toutes les interactions subies par les ions, quelques unes se produisent à très petit paramètre d'impact (inférieur à $10^{-4} \mathrm{~nm}$ ). Les événements qui peuvent se produire dans ce cas sont de plusieurs types : déflexions élastiques à grand angle ou réactions nucléaires. Les ionisations en couche interne ont une section efficace importante dès que le paramètre d'impact est inférieur à $10^{-2} \mathrm{~nm}$.

Ce sont ces événements qui vont être utilisés pour la microanalyse nucléaire, par détection et analyse en énergie des ions rétrodiffusés ou des ions de recul lors des déflexions élastiques (RBS, ERDA), des particules et/ou des photons $\gamma$ émis lors des réactions nucléaires (NRA) ou des photons $\mathrm{X}$ émis suite aux ionisations en couche interne des atomes de la cible (PIXE).

\subsubsection{Les différentes techniques de microanalyse nucléaire}

Les techniques de microanalyse correspondantes sont respectivement :

- la spectrométrie des ions rétrodiffusés élastiquement (RBS : Rutherford Backscattering Spectrometry),

- la spectrométrie des atomes de recul issus d'une collision élastique (ERDA, Elastic Recoil

Detection Analysis, ou FRES, Forward Recoil Elastic Spectrometry),

- l'analyse par réactions nucléaires (NRA: Nuclear Reaction Analysis),

- la spectrométrie des photons X (PIXE: Particle Induced X-ray Emission). 
Les détecteurs les plus couramment utilisés pour compter et analyser en énergie les particules ou les photons émis sont des dispositifs semi-conducteurs. Pour les particules il s'agit de diodes à barrière de surface polarisées en inverse où les particules perdent toute leur énergie dans la zone désertée créant un nombre de paires électrons-trous en nombre proportionnel à l'énergie déposée ; la résolution (largeur à mi-hauteur FWHM) de la chaîne d'analyse (détecteur, électronique de mise en forme et d'amplification, convertisseur analogique-numérique, analyseur multicanaux) est autour de $15 \mathrm{keV}$.

Pour les photons $\mathrm{X}$, on utilise le même principe, mais avec un dispositif particulier (silicium quasi-intrinsèque dopé au lithium près de la surface, refroidi et suffisamment épais pour arrêter tous les photons $\mathrm{X}$ d'intérêt). On obtient dans ce cas des résolutions en énergie inférieures à $200 \mathrm{eV}$, ce qui permet de bien séparer les pics correspondant aux différentes transitions dans les différents atomes à condition que ceux-ci ne soient pas de $Z$ trop bas.

Pour les photons $\gamma$, on utilise soit un cristal scintillateur accolé à un photomultiplicateur, soit un gros cristal de germanium intrinsèque qui contrairement au précédent permet d'obtenir une très bonne résolution en énergie, mais qui n'a une efficacité de détection que de 20 à $40 \%$.

\section{SPECTROMÉTRIE DES COLLISIONS ÉLASTIQUES À PETIT PARAMÈTRE D'IMPACT}

\subsection{Spectrométrie des ions rétrodiffusés : RBS}

Dans cette technique, la plus largement utilisée, les ions diffusés élastiquement vers l'arrière sont comptés et analysés en énergie. L'angle de détection dans le référentiel du laboratoire, par rapport à la direction du faisceau incident, $\theta_{l a b}$ est fixé à une valeur supérieure à $90^{\circ}$.

\subsubsection{Cinématique, analyse qualitative}

L'énergie d'un ion diffusé est fonction de sa masse, de l'angle de détection, de la masse du noyau diffuseur et est proportionnelle à l'énergie des ions du faisceau incident : $E_{d}=K E_{o}, K=f\left(M_{1}, M_{2}, \theta_{l a b}\right)$ est donné simplement par les conservations de l'énergie cinétique et de la quantité de mouvement. L'expression de $K$ est :

$$
K=\left[\frac{\left(M_{2}{ }^{2}-M_{1}{ }^{2} \sin ^{2} \theta\right)^{1 / 2}+M_{1} \cos \theta}{M_{1}+M_{2}}\right] .
$$

$K$ croît quand la masse $M_{2}$ de l'atome cible croît pour un ion incident donné de masse $M_{1}$ ( $\underline{\text { Fig. } 3}$ ).

Une rétrodiffusion $\left(\theta_{l a b}>90^{\circ}\right)$ ne peut évidemment avoir lieu si l'atome cible a une masse inférieure à celle de l'ion incident. En considérant le cas limite $\theta_{l a b}=180^{\circ}$, on obtient :

$$
K=\frac{\left(M_{2}-M_{1}\right)^{2}}{\left(M_{2}+M_{1}\right)^{2}} .
$$

À $E_{o}, M_{1}$ et $\theta_{l a b}$ fixés, l'énergie d'un ion rétrodiffusé est donc caractéristique de la masse de l'atome cible présent dans l'échantillon, ce qui permet de caractériser sa nature. Sur la figure 4 est représenté le spectre en énergie idéal des ions ${ }^{4} \mathrm{He}$, initialement d'une énergie de $2 \mathrm{MeV}$, rétrodiffusés par une couche théorique ultra-mince (monocouche) multi-éléments sans support. 


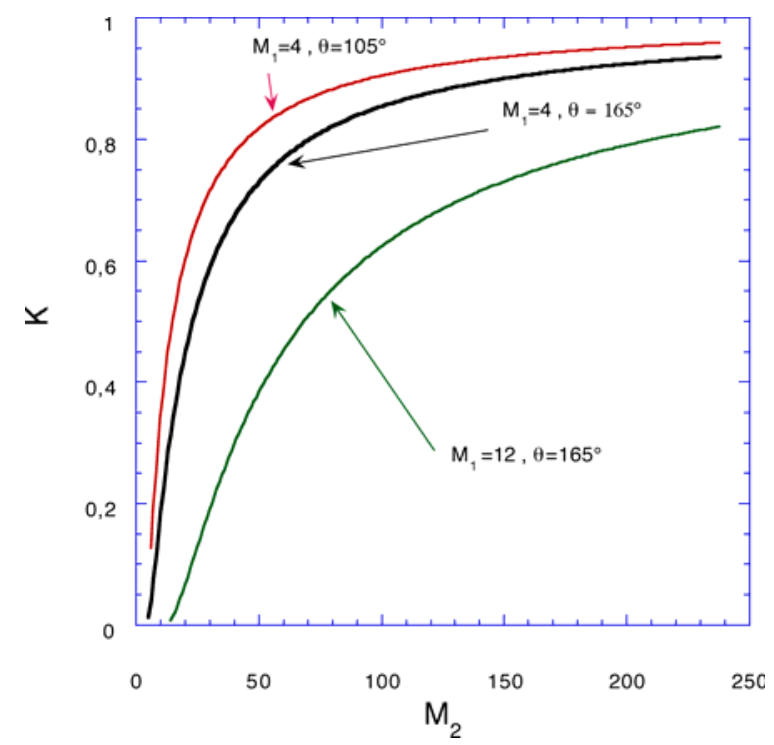

FIG. 3. Exemples de variation du coefficient $K$ de rétrodiffusion élastique avec la masse de l'atome cible, l'angle de diffusion et la masse de l'ion incident.

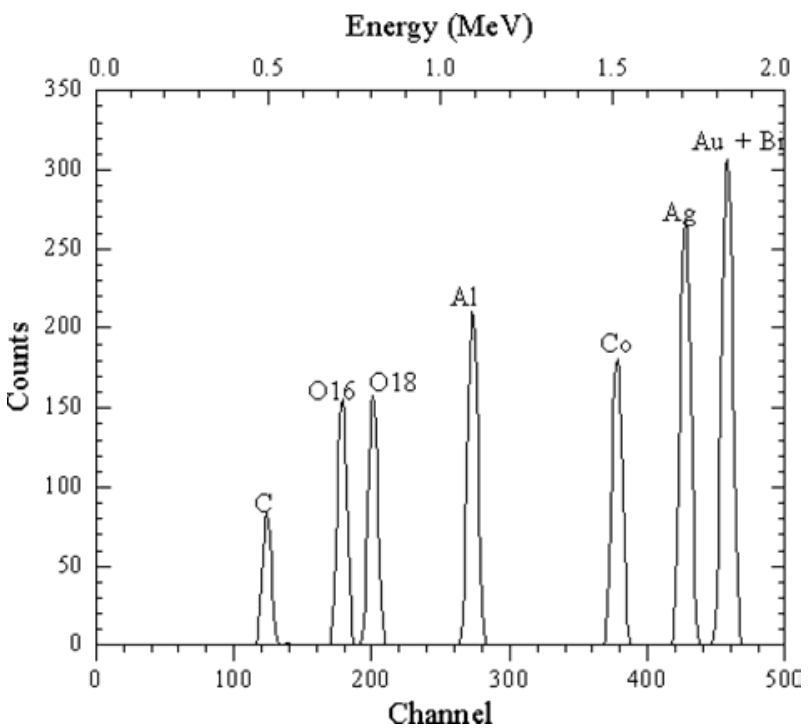

FIG. 4. Simulation d'un spectre RBS d'une couche ultra-mince multi-éléments.

La largeur des pics est due à la résolution instrumentale. Il est mis en évidence que la résolution en masse diminue quand la masse de l'atome cible augmente : si l'on peut distinguer les différents isotopes des éléments légers, on ne peut distinguer les pics provenant des éléments très lourds de masses voisines.

Considérons maintenant un échantillon comportant une couche très mince sur un substrat comportant des éléments plus légers que ceux contenus dans la couche. Les ions rétrodiffusés par la couche mince lourde ont une énergie plus grande que ceux qui sont rétrodiffusés sur le substrat léger et leur spectre en énergie est un pic dont la largeur est proche de la résolution instrumentale en énergie. 


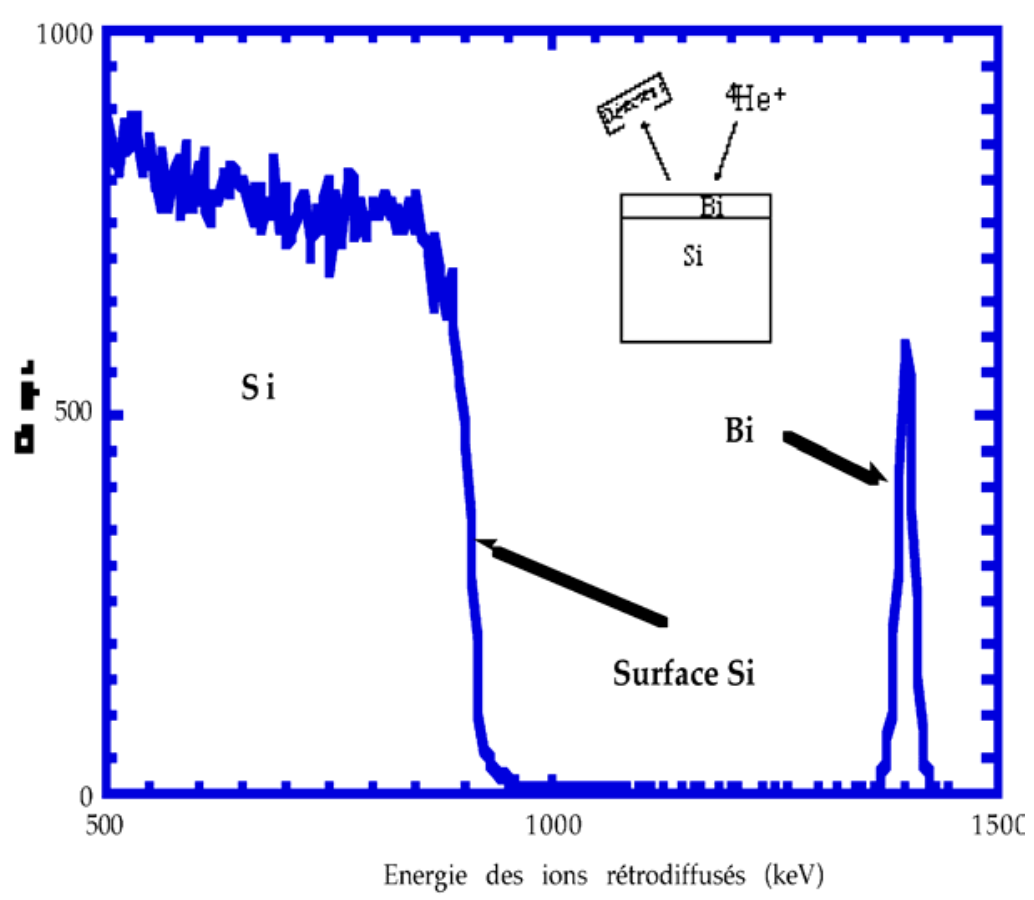

FIG. 5. Spectre RBS d'une cible composée de $5.10^{15}$ atomes de bismuth sur silicium massif.

La partie du spectre correspondant aux ions rétrodiffusés par les atomes du substrat est continue et s'étend jusqu'à $E_{d}=0$. En effet, tout au long de leur parcours, les ions incidents peuvent être rétrodiffusés mais à une profondeur $x$, l'ion incident aura perdu de l'énergie lors de son trajet aller dans la matière et reperdra de l'énergie sur son parcours de sortie avant d'être détecté. Les ions rétrodiffusés sur les premières couches du substrat seront détectés à une énergie inférieure à $K_{S} E_{O}$ à cause des pertes d'énergie au passage de la couche mince.

\subsection{2. Échelle des profondeurs}

De manière générale, l'énergie d'un ion détecté va être non seulement fonction de la nature de l'atome diffuseur mais aussi de la quantité de matière, ainsi que la nature des atomes qui le séparent de la surface. On peut exprimer cela par l'expression $E_{d}=K\left(E_{o}-\Delta E_{\text {in }}\right)-\Delta E_{\text {out }}$, où $K$ est le coefficient correspondant à l'atome diffuseur, $\Delta E_{\text {in }}$ et $\Delta E_{\text {out }}$ les pertes d'énergie sur les parcours aller et retour respectivement (Fig. 6). Les pertes d'énergie sont déterminées par les pouvoirs d'arrêt des atomes situés entre l'atome diffuseur et la surface, ainsi que par la géométrie de l'expérience.

Cela permet de faire, dans une cible donnée, une correspondance biunivoque entre $E_{d}$ et la profondeur $x$ de rétrodiffusion en valeurs moyennes à cause de la résolution et des effets de straggling, élément par élément, et ainsi de pouvoir déterminer les profils de concentration des différents éléments présents dans l'échantillon. 


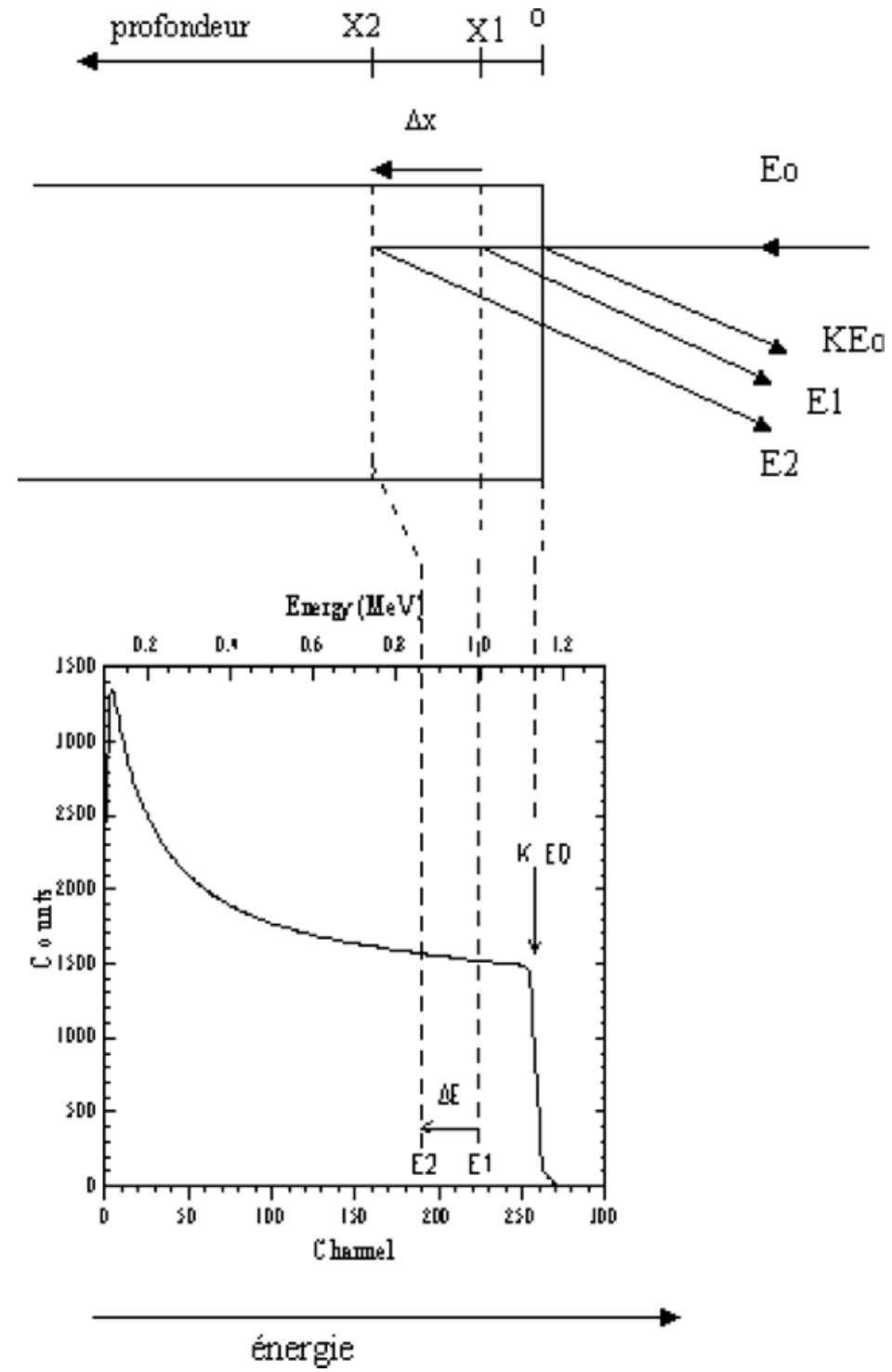

FIG. 6. Principe de la correspondance énergie détectée - profondeur de réaction.

\subsubsection{Section efficace de rétrodiffusion. Analyse quantitative.}

La section efficace différentielle de rétrodiffusion élastique pour l'interaction entre deux charges ponctuelles est donnée par la formule de Rutherford:

$$
\frac{\mathrm{d} \sigma}{\mathrm{d} \Omega}=\left\{\frac{Z_{1} Z_{2} e^{2}}{4 E}\right\}^{2} \frac{1}{\sin ^{4} \frac{\theta}{2}},
$$

où $\theta$ est l'angle de diffusion dans le système du centre de masse et $E$ l'énergie dans le système du laboratoire, $Z_{1}$ et $Z_{2}$ étant respectivement les charges des noyaux de l'ion incident et de l'atome cible. Il est important de remarquer la dépendance en $Z_{2}^{2}$ qui indique la très grande sensibilité aux éléments lourds. Il faut apporter un terme correctif d'écrantage par les électrons, non négligeable aux basses vitesses et pour les atomes de $Z$ élevé. Ce terme d'écrantage est bien représenté par :

on a donc $\left[\frac{\mathrm{d} \sigma}{\mathrm{d} \Omega}\right]_{\text {vrai }}=F\left[\frac{\mathrm{d} \sigma}{\mathrm{d} \Omega}\right]_{\text {Ruth. }}$.

$$
F=1-\frac{0,049 Z_{1} Z_{2}^{4 / 3}}{E} ;
$$


Aux grandes vitesses, la distance minimum d'approche est suffisamment petite pour que les forces d'interaction nucléaire interviennent. On observe alors des écarts importants à la section efficace Rutherford (Fig. 7).
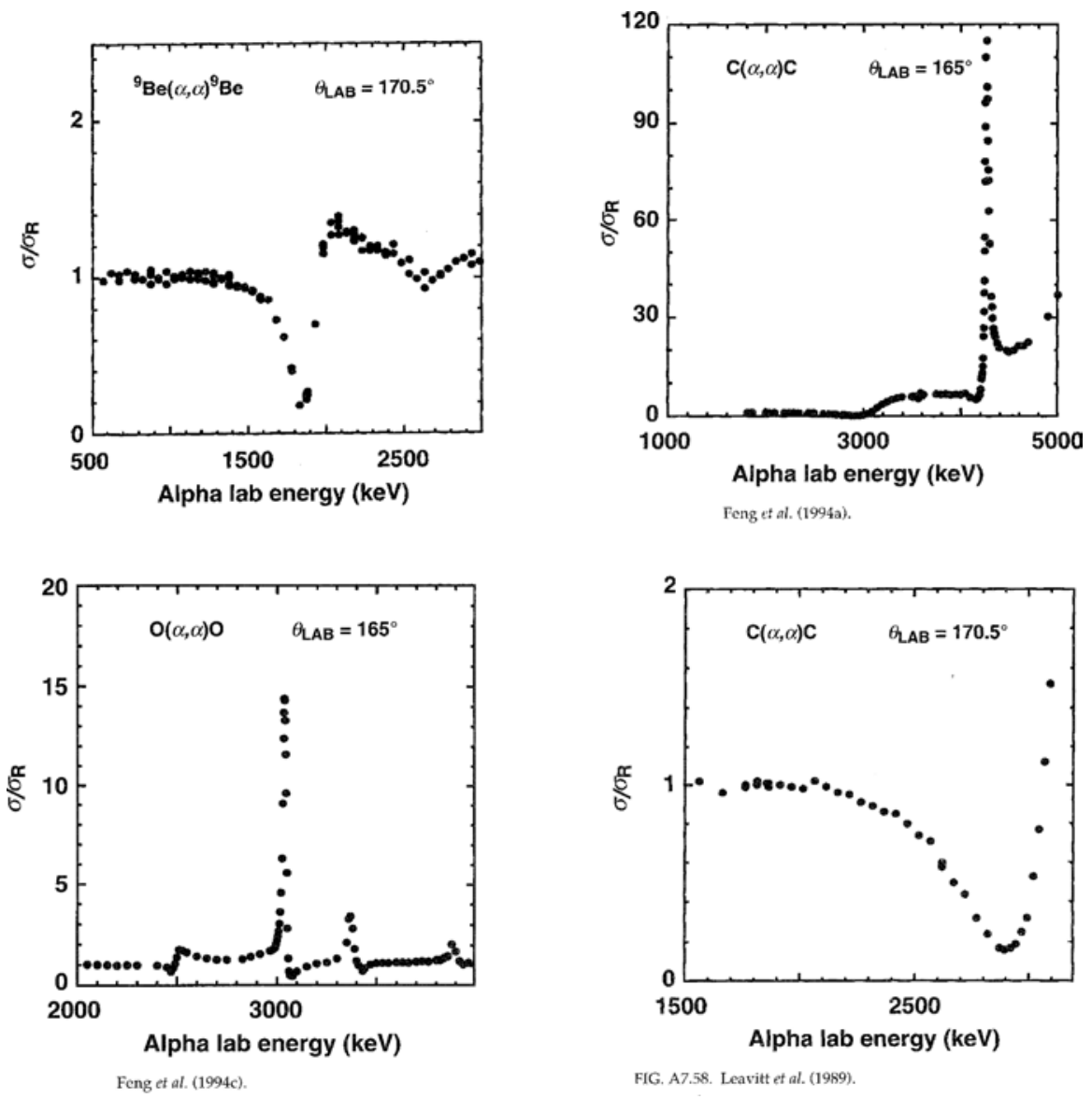

FIG. 7. Quelques exemples d'écarts de sections efficaces de diffusion élastique à la loi de Rutherford. En ordonnée est porté le rapport de la section efficace vraie à la section efficace donnée par la loi de Rutherford.

\subsubsection{Spectres en énergie des ions rétrodiffusés : $N\left(E_{d}\right)$}

Un spectre de rétrodiffusion élastique peut s'exprimer par

$$
N_{i}(E) \delta E=Q C_{i}(x) d x \frac{\mathrm{d} \sigma_{i}(\mathrm{E}(\mathrm{x}))}{\mathrm{d} \Omega} \Delta \Omega,
$$

où $N_{i}\left(E_{d}\right) \delta E_{d}$ est le nombre de coups dans l'intervalle d'énergie ( $\left.E_{d}, E_{d}+\delta E_{d}\right)$, correspondant à l'élément $\mathrm{n}^{\circ} i$ dans l'épaisseur $\mathrm{d} x$ à la profondeur $x, \delta E$ correspondant à l'intervalle de profondeur $\mathrm{d} x$, via la relation $E(x)$. $Q$ est le nombre d'ions incidents, $C_{i}(x)$ le nombre d'atomes par $\mathrm{cm}^{3}$ de l'espèce $i$ à la profondeur $x, \frac{\mathrm{d} \sigma_{i}(E(x))}{\mathrm{d} \Omega}$ la section efficace pour l'espèce $i$ pour l'énergie de l'ion à la profondeur $x$, $\Delta \Omega$ étant l'angle solide de détection. Ceci est l'expression pour chaque élément présent dans la cible, le spectre mesuré est la somme des différents spectres pour chacun des éléments (Fig. 8). On remarquera que le comptage $N_{i}(E)$ qui est le nombre de coups par unité d'énergie détectée est proportionnel à l'inverse de la dérivée de la relation $E_{d}(x)$. 

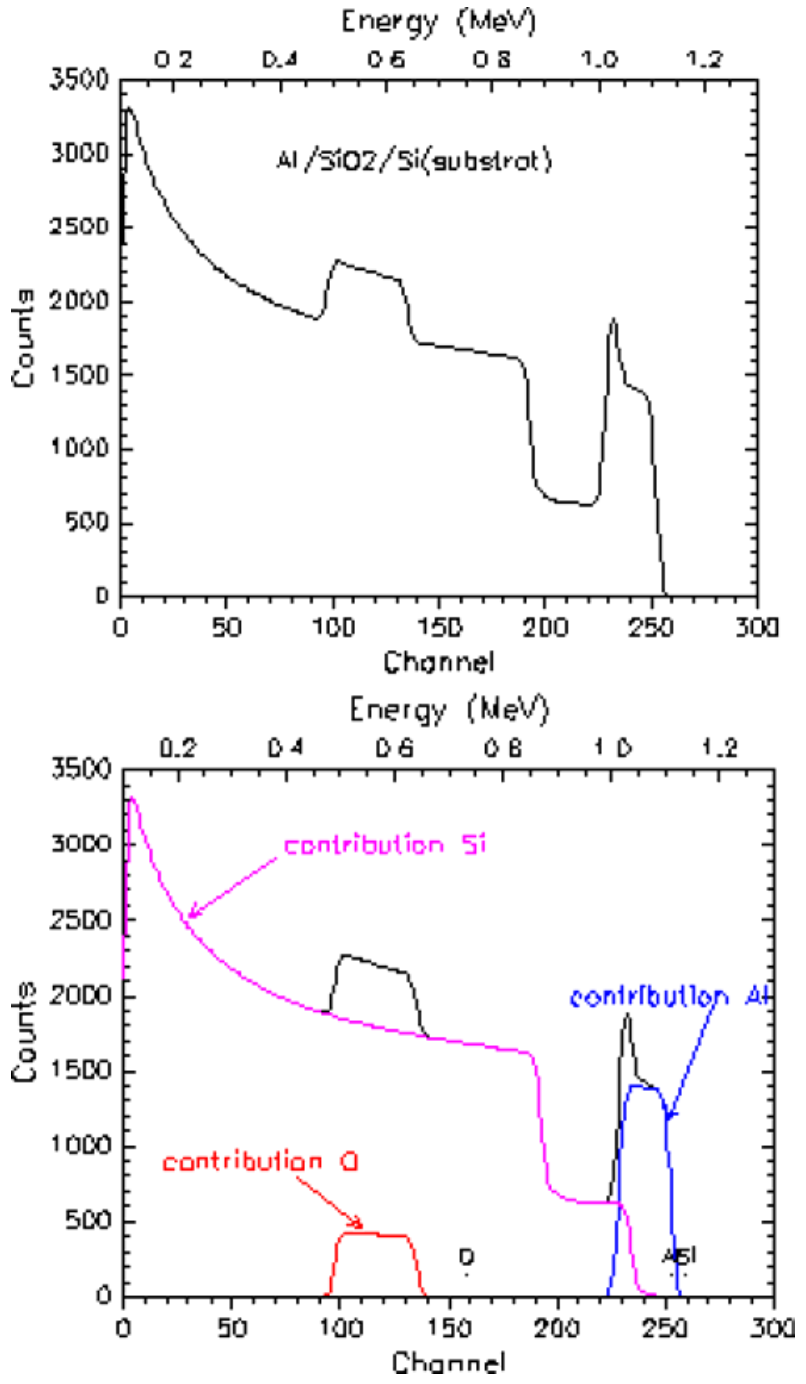

FIG. 8. Simulation du spectre RBS d'une couche mince d'aluminium déposée sur du silicium oxydé superficiellement. En bas de la figure sont montrées les contributions au spectre des différents éléments présents dans la cible.

Pour une couche mince, l'expression est simplifiée si l'on peut négliger la variation de section efficace à la traversée de la couche par les ions incidents : l'intégrale du pic est

$$
\int N_{i}(E) \delta E=n_{i} Q \frac{\partial \sigma}{\mathrm{d} \Omega} \Delta \Omega,
$$

où $n_{i}$ le nombre total d'atomes de l'espèce $i$ dans la couche.

Dans cette expression, la quantité $Q$ d'ions incidents (dose) est aisément mesurée. Par contre, il est difficile de déterminer avec une grande précision l'angle solide de détection $\Delta \Omega$. C'est pourquoi, on utilise une cible mince de référence (couramment du Bi implanté à très faible profondeur dans du silicium) où un élément lourd est en quantité bien connue. Ainsi la détermination quantitative d'un élément sera pour les mêmes conditions d'analyse pour la référence et l'échantillon :

$$
n_{i}=n_{\text {réf }} \frac{N_{i}}{N_{\text {réf }}} \frac{\left[\frac{\partial \sigma}{\mathrm{d} \Omega}\right]_{r e f}}{\left[\frac{\partial \sigma}{\mathrm{d} \Omega}\right]_{i}} .
$$




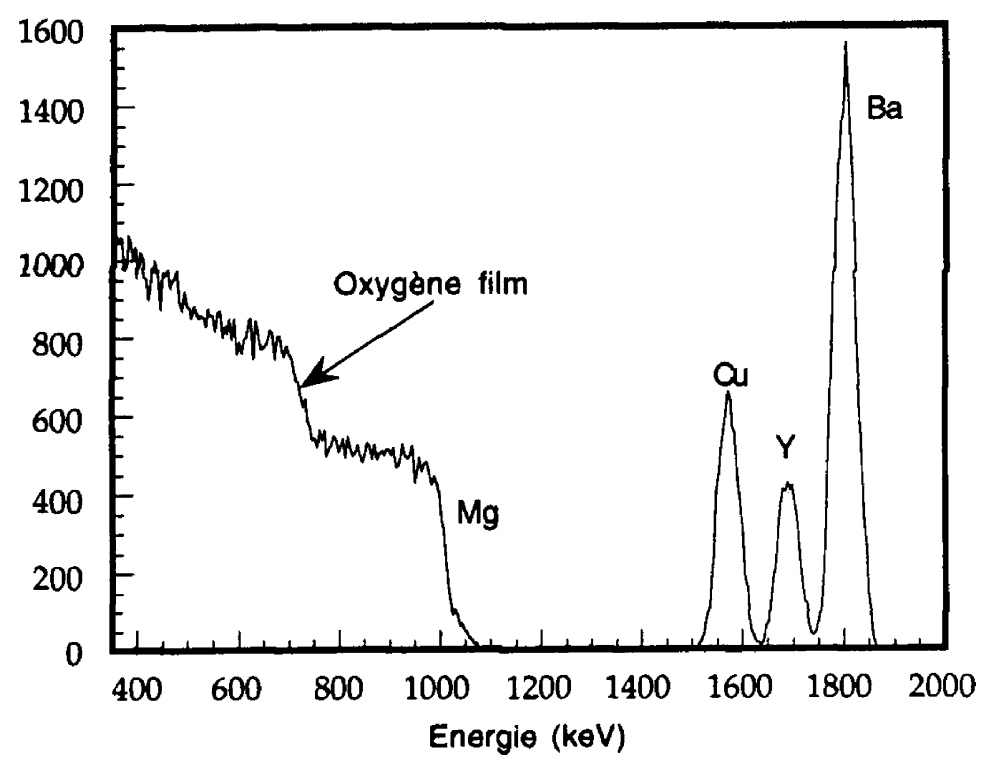

FIG. 9. Spectre RBS d'un film mince $\mathrm{YBa}_{2} \mathrm{Cu}_{3} \mathrm{O}_{7-\delta}$ déposé sur $\mathrm{MgO}$ massif : détermination de la stœchiométrie cationique.

\subsubsection{Profils de concentration}

Comme on l'a vu plus haut, l'échelle d'énergie peut être convertie en échelle de profondeur pour chacun des éléments présents dans la cible. Chaque composante élémentaire d'un spectre est ainsi une image du profil de concentration de chaque élément dans l'échantillon.

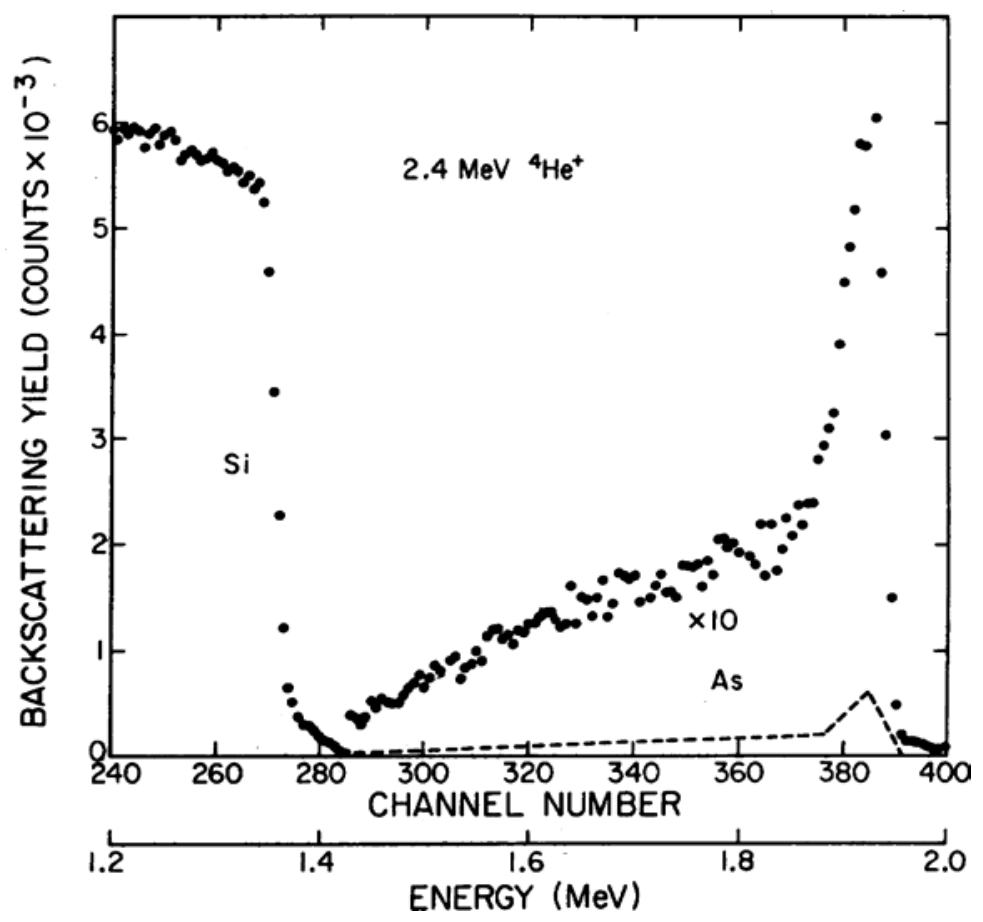

FIG. 10. Spectre d'un échantillon de silicium implanté en arsenic et recuit : détermination du profil de concentration de l'arsenic. 


\subsubsection{Sensibilité et résolution}

À cause des propriétés ci-dessus (section efficace en $Z_{2}^{2}$ et $K$ croissant avec $M_{2}$ ), la RBS est particulièrement indiquée pour le dosage des couches minces formées d'éléments lourds sur des substrats légers. Dans ces cas on peut atteindre une sensibilité nettement inférieure à la monocouche atomique. Mais la résolution en masse se dégrade quand la masse croît. On ne pourra donc différencier deux éléments lourds de masse élevées et très proches. Par contre dans la partie du spectre correspondant aux éléments légers, si les composantes lourdes sont uniquement en couche mince, on pourra même différencier les isotopes d'un même élément.

La résolution en profondeur des profils de concentration est variable avec la nature des cibles. Cette résolution s'améliore quand le pouvoir d'arrêt augmente, c'est à dire pour des éléments lourds. Elle peut atteindre moins de $10 \mathrm{~nm}$ dans les cas favorables près de la surface ; mais sa dégradation est inévitable quand la profondeur analysée augmente, du fait de la dispersion en énergie et géométrique des ions à la traversée de la matière.

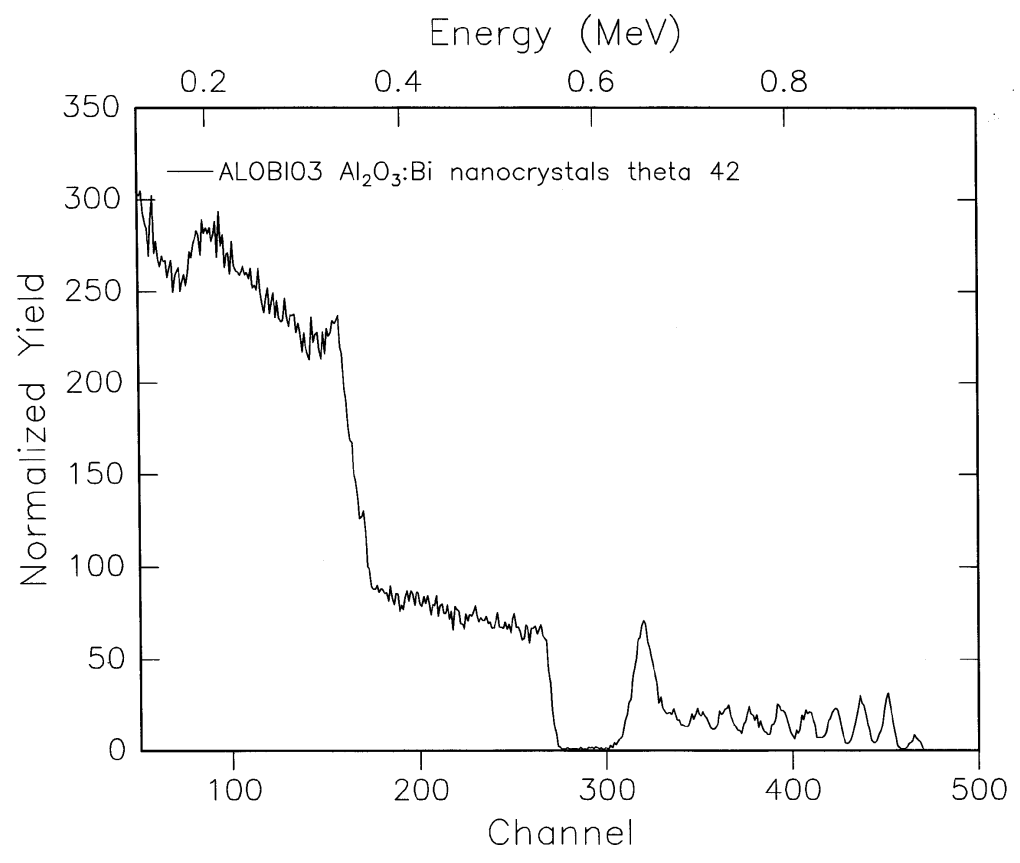

FIG. 11. Spectre RBS d'une structure multicouches de nanocristaux de $\mathrm{Bi}$ et $\mathrm{Cu}$ dans $\mathrm{Al}_{2} \mathrm{O}_{3}$ formée par ablation laser. La partie haute énergie du spectre correspond à la succession de 10 couches de $\mathrm{Bi}$ d'environ $10 \mathrm{~nm}$ chacune, séparées par la même épaisseur d' $\mathrm{Al}_{2} \mathrm{O}_{3}$.

\subsection{Analyse par détection des ions de recul élastique (ERDA)}

Dans cette technique, on analyse en énergie non pas les ions incidents rétrodiffusés, mais les atomes cible de recul lors des collisions élastiques à faible paramètre d'impact. Pour cela, on utilise une géométrie de détection vers l'avant, le faisceau d'analyse étant en incidence rasante.

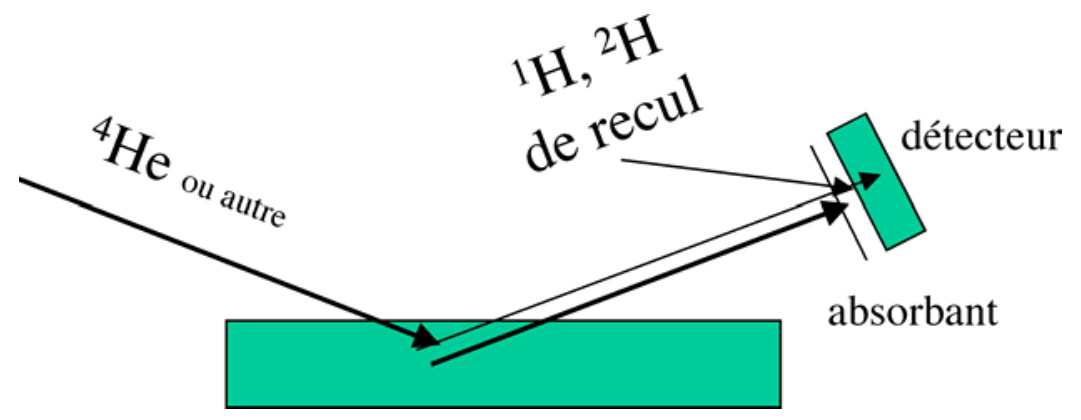

FIG. 12. Principe des expériences de mesures par ERDA. 


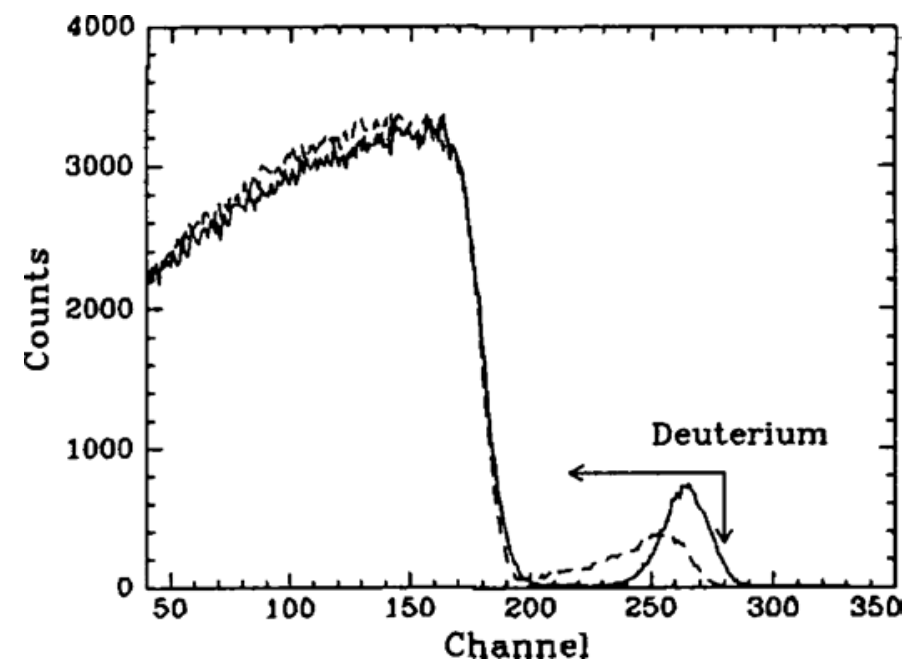

FIG. 13. Spectre ERDA d'une couche de polystyrène deutéré sur du polystyrène non deutéré avant (ligne continue) et après (ligne discontinue) interdiffusion.
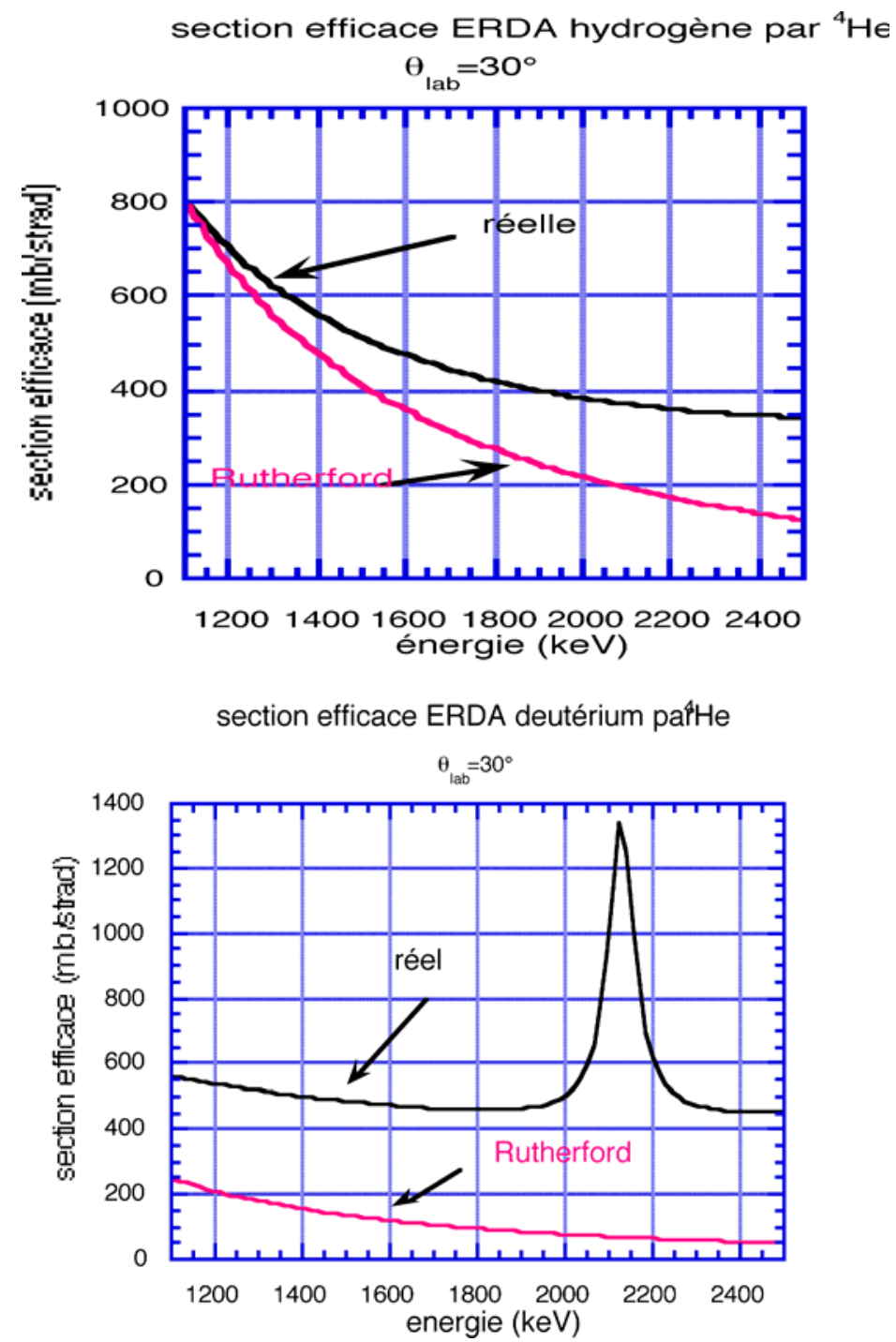

FIG. 14. Sections efficaces de recul élastique à $\theta_{l a b}=30^{\circ}$ pour ${ }^{4} \mathrm{He}$ sur $\mathrm{H}$ (haut) et ${ }^{2} \mathrm{H}$ (bas). Les sections efficaces calculées par la loi de Rutherford sont figurées sur chaque graphe. 
La section efficace de ces collisions est supérieure à celle que l'on peut attendre de simples collisions élastiques de type Rutherford (Fig. 14). En effet, il s'agit de la collision entre deux atomes de faible $Z$, la distance minimum d'approche est très petite et les interactions nucléaires interviennent.

La sensibilité est donc très bonne, typiquement inférieure à la monocouche. Mais deux phénomènes compliquent l'analyse :

a) il est souvent observé une désorption d'hydrogène de la couche sous le faisceau d'analyse. Il faudra donc faire une étude de cette éventuelle désorption et utiliser de faibles doses d'ions incidents par point d'analyse.

b) tout échantillon ayant « vu» l'atmosphère va être pollué. On risque donc, en particulier si la quantité d'hydrogène à mesurer est faible, de doser une couche de pollution et non l'hydrogène dans la couche. Le meilleur moyen de s'affranchir de cela est d'utiliser le traçage isotopique au deutérium.

Comme pour la RBS, il est important de disposer d'une cible référence d'hydrogène et/ou de deutérium de composition bien connue.

\section{UTILISATION DES RÉACTIONS NUCLÉAIRES (NRA)}

Quand le paramètre d'impact d'un ion léger incident ( $\mathrm{p}, \mathrm{d},{ }^{3} \mathrm{He}$ ) sur un noyau léger $\left(Z_{2}<16\right.$ aux énergies de l'ordre du $\mathrm{MeV}$ considérées ici) est très petit, il peut se produire une réaction nucléaire avec émission dans un temps très court $\left(10^{-16}\right.$ à $\left.10^{-18} \mathrm{~s}\right)$ d'une particule et/ou d'un photon $\gamma$. On peut considérer qu'il s'agit de la formation d'un noyau composé instable qui se décompose en un noyau résiduel et une particule que l'on détecte. Il s'agit cette fois d'une réaction inélastique, la différence des masses avant et après réaction correspondant au « $Q$ » de la réaction. Cette valeur $Q$ est caractéristique de la réaction. La nature et l'énergie de la particule émise vont donc caractériser la réaction et donc le noyau de la cible qui a réagi.

On a ici non seulement la possibilité d'analyser les éléments mais aussi les isotopes d'un même élément, ce qui va permettre des expériences de traçage isotopique, par exemple :

$$
\begin{aligned}
& { }^{16} \mathrm{O}+\mathrm{d} \rightarrow{ }^{17} \mathrm{O}+\mathrm{p}\left(Q_{o}=1,919 \mathrm{MeV}, Q_{1}=1,046 \mathrm{MeV}\right) \\
& \text { et }{ }^{18} \mathrm{O}+\mathrm{p} \rightarrow{ }^{15} \mathrm{~N}+\alpha(Q=3,970 \mathrm{MeV})
\end{aligned}
$$

en abrégé ${ }^{16} \mathrm{O}(\mathrm{d}, \mathrm{p})^{17} \mathrm{O}$ et ${ }^{18} \mathrm{O}(\mathrm{p}, \alpha){ }^{15} \mathrm{~N}$. De même pour ${ }^{14} \mathrm{~N}(\mathrm{~d}, \alpha){ }^{12} \mathrm{C}$ et ${ }^{15} \mathrm{~N}(\mathrm{~d}, \alpha)^{13} \mathrm{C}$.

Pour une même réaction, il peut apparaître différents pics, correspondant aux différents états excités du noyau résiduel, la valeur $Q$ diminuant avec le degré d'excitation du noyau résiduel.

Dans le tableau suivant, des exemples de réactions nucléaires utilisées en microanalyse nucléaire sont mentionnées. Les valeurs $Q$ indiquées correspondent à l'état fondamental du noyau résiduel, sauf mention particulière : par exemple ${ }^{14} \mathrm{~N}\left(\mathrm{p}_{5}\right)$ correspond à la valeur $Q$ de la réaction si le noyau résiduel ${ }^{15} \mathrm{~N}$ est dans son cinquième état excité. 


\begin{tabular}{|c|c|c|c|c|c|}
\hline \multicolumn{2}{|c|}{ Réactions $\mathrm{X}(\mathbf{p}, \alpha) \mathrm{Y}$} & \multicolumn{2}{|c|}{ Réactions $X(d, \alpha) Y$} & \multicolumn{2}{|c|}{ Réactions X $(\mathbf{d}, \mathbf{p}) \mathrm{Y}$} \\
\hline Isotope X & $Q(\mathrm{MeV})$ & Isotope $\mathrm{X}$ & $Q(\mathrm{MeV})$ & Isotope X & $Q(\mathrm{MeV})$ \\
\hline${ }^{6} \mathrm{Li}$ & 4,020 & ${ }^{6} \mathrm{Li}$ & 22,36 & ${ }^{6} \mathrm{Li}$ & 5,027 \\
\hline${ }^{7} \mathrm{Li}$ & 17,347 & ${ }^{7} \mathrm{Li}$ & 14,163 & ${ }^{9} \mathrm{Be}$ & 4,585 \\
\hline${ }^{9} \mathrm{Be}$ & 2,125 & ${ }^{9} \mathrm{Be}$ & 7,152 & ${ }^{10} \mathrm{~B}$ & 9,237 \\
\hline${ }^{10} \mathrm{~B}$ & 1,147 & ${ }^{10} \mathrm{~B}$ & 17,819 & ${ }^{11} \mathrm{~B}$ & 1,138 \\
\hline${ }^{11} \mathrm{~B}$ & 8,582 & ${ }^{11} \mathrm{~B}$ & 8,022 & ${ }^{12} \mathrm{C}$ & 2,719 \\
\hline${ }^{15} \mathrm{~N}$ & 4,964 & ${ }^{13} \mathrm{C}$ & 5,167 & ${ }^{13} \mathrm{C}$ & 5,947 \\
\hline${ }^{17} \mathrm{O}$ & 1,197 & ${ }^{14} \mathrm{~N}$ & 13,579 & ${ }^{14} \mathrm{~N}$ & 8,615 \\
\hline${ }^{18} \mathrm{O}$ & 3,970 & ${ }^{14} \mathrm{~N}\left(\alpha_{1}\right)$ & 9,146 & ${ }^{14} \mathrm{~N}\left(\mathrm{p}_{5}\right)$ & 1,305 \\
\hline${ }^{19} \mathrm{~F}$ & 8,119 & ${ }^{15} \mathrm{~N}$ & 7,983 & ${ }^{15} \mathrm{~N}$ & 0,267 \\
\hline${ }^{23} \mathrm{Na}$ & 2,379 & ${ }^{16} \mathrm{O}$ & 3,116 & ${ }^{16} \mathrm{O}\left(\mathrm{p}_{0}\right)$ & 1,919 \\
\hline \multirow[t]{7}{*}{${ }^{27} \mathrm{Al}$} & 1,594 & ${ }^{17} \mathrm{O}$ & 9,812 & ${ }^{16} \mathrm{O}\left(\mathrm{p}_{1}\right)$ & 1,046 \\
\hline & & ${ }^{18} \mathrm{O}$ & 4,237 & ${ }^{18} \mathrm{O}$ & 1,731 \\
\hline & & ${ }^{19} \mathrm{~F}$ & 10,038 & ${ }^{19} \mathrm{~F}$ & 4,379 \\
\hline & & ${ }^{23} \mathrm{Na}$ & 6,909 & ${ }^{23} \mathrm{Na}$ & 4,734 \\
\hline & & ${ }^{24} \mathrm{Mg}$ & 1,964 & ${ }^{24} \mathrm{Mg}$ & 5,106 \\
\hline & & ${ }^{27} \mathrm{Al}$ & 6,701 & ${ }^{27} \mathrm{Al}$ & 5,499 \\
\hline & & ${ }^{28} \mathrm{Si}$ & 1,421 & ${ }^{28} \mathrm{Si}$ & 6,253 \\
\hline
\end{tabular}

D'autres possibilités sont aussi utilisées. Par exemple, les faisceaux d'3 ${ }^{3} \mathrm{He}$ permettent aussi, par utilisation de réactions $\left({ }^{3} \mathrm{He}, \mathrm{p}\right)$ ou $\left({ }^{3} \mathrm{He}, \alpha\right)$, le dosage de ${ }^{9} \mathrm{Be},{ }^{11} \mathrm{~B},{ }^{14} \mathrm{~N},{ }^{16} \mathrm{O}$.

Quand on peut disposer de faisceau d'ions plus lourds et de plus haute énergie (jusqu'à une dizaine de $\mathrm{MeV})$ on peut utiliser pour doser les éléments très légers $\left(\mathrm{H},{ }^{2} \mathrm{H}\right.$ par exemple) des réactions inversées où l'on utilise comme particule incidente l'atome cible des réactions ci-dessus. Pour être dans des conditions équivalentes, il faut alors travailler à une énergie plus grande d'un facteur égal au rapport des masses cible/ion incident. À titre d'exemple, citons ${ }^{1} \mathrm{H}\left({ }^{15} \mathrm{~N}, \alpha \gamma\right)$.

\subsection{Analyses par étude du spectre en énergie des particules émises}

De même qu'avec la RBS, on obtient une analyse qualitative et quantitative et une possibilité de détermination de profil de concentration, mais dans cette technique seuls les éléments pouvant donner une réaction nucléaire dans les conditions expérimentales choisies vont apparaître. On obtient donc une sélectivité isotopique de l'analyse, au contraire de la RBS où tous les éléments présents peuvent donner lieu à rétrodiffusion (sauf si leur masse est inférieure à la masse de l'ion incident). Afin de ne détecter que les produits de réactions nucléaires, on interpose une feuille mince d'absorbant, choisie de manière à arrêter les ions rétrodiffusés. 


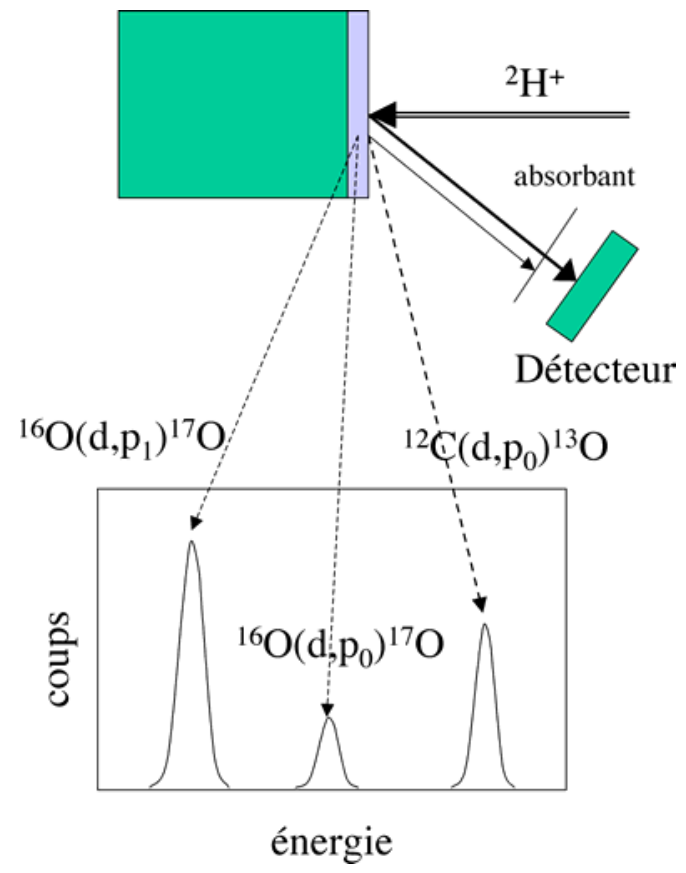

FIG. 15. Exemple du dosage de l'oxygène dans une couche mince d'oxyde sur substrat lourd. Le pic carbone provient de la pollution de surface.

Lors du choix des conditions expérimentales on va rechercher évidemment une valeur suffisamment grande de la section efficace, mais aussi une géométrie de détection et une énergie de faisceau qui permettent d'obtenir des informations claires. Par exemple, quand il y a possibilité de superposition de pics correspondant à des réactions sur des éléments différents, l'ajustement de divers paramètres de l'analyse permet dans la très grande majorité des cas d'isoler un ou plusieurs pics caractéristiques de l'élément d'intérêt. Le choix de la particule incidente, de l'énergie et de l'angle de détection permet par exemple de se placer dans des conditions où une réaction gênante a une section efficace négligeable ou bien où l'énergie des particules émises est telle que les pics sont bien séparés. Le choix de l'épaisseur d'absorbant et/ou du détecteur va permettre, par exemple, de séparer un pic de particules $\alpha$ d'un pic de protons en utilisant le fait que les $\alpha$ sont plus rapidement freinés que les protons.

Pour la détermination des profils de concentration, par rapport à la technique RBS, l'analyse est compliquée par la forme non simple des sections efficaces.

Pour l'analyse de couches minces, le cas favorable est, à l'opposé de la RBS, celui des éléments légers sur un substrat lourd qui ne produira pas de réactions. Pour simplifier le dépouillement, on se placera à une énergie où la section efficace varie peu avec l'énergie de manière à ce que le rendement de la réaction ne soit pas dépendante de la profondeur d'analyse (Fig. 16). De même que dans le cas des techniques précédemment décrites, il est nécessaire de disposer d'échantillons de référence contenant une quantité bien connue de l'isotope analysé. 
sections efficaces: $12 \mathrm{C}(\mathrm{d}, \mathrm{p})$ et $160(\mathrm{~d}, \mathrm{p} 1) 135^{\circ}$ et $150^{\circ}$

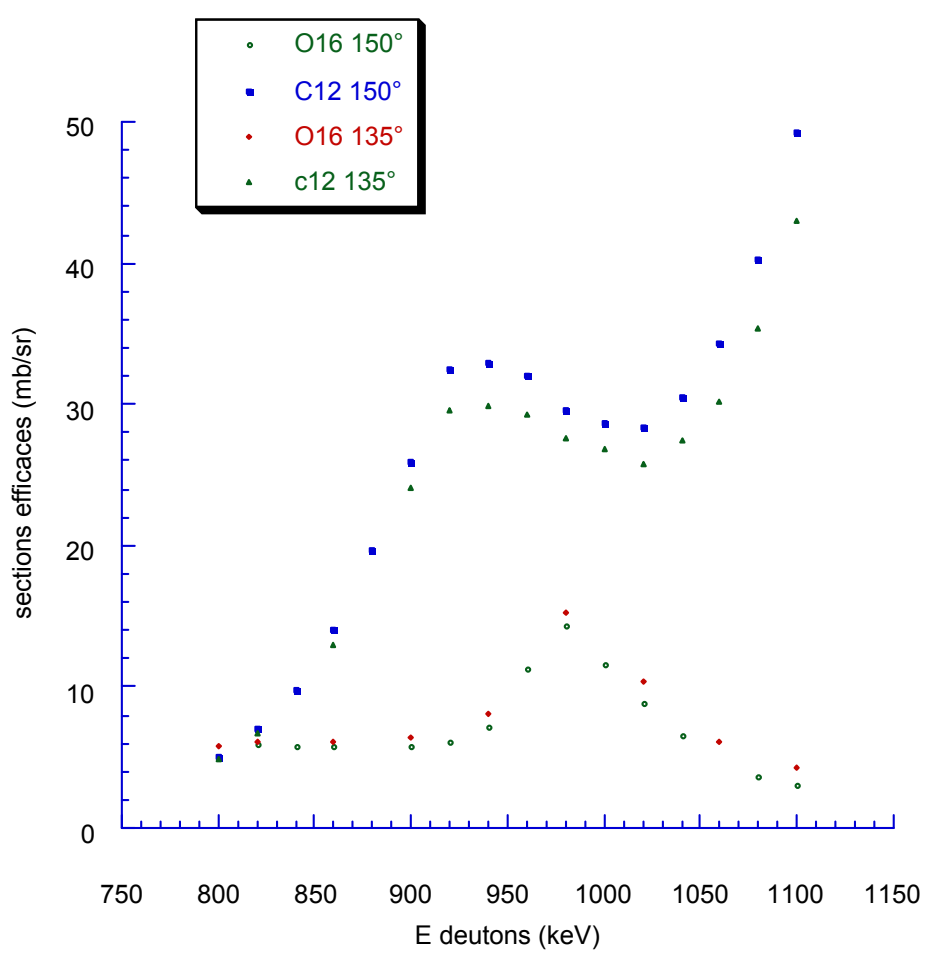

FIG. 16. Exemples de sections efficaces. Pour le dosage, on choisit des régions où la section efficace varie peu. Le dosage de l'oxygène est effectué entre 900 et $800 \mathrm{keV}$, le dosage du carbone entre $1 \mathrm{MeV}$ et $950 \mathrm{keV}$.
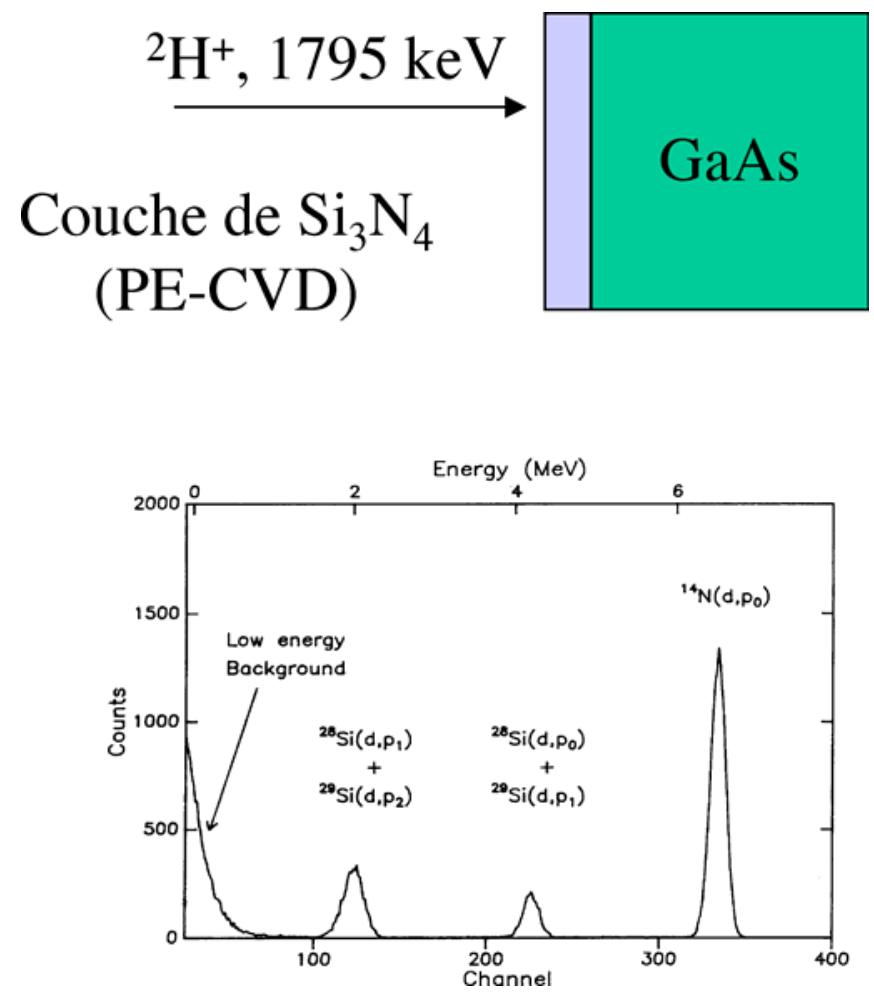

FIG. 17. Détermination de la stœchiométrie d'une couche de nitrure de silicium déposée sur GaAs par utilisation des réactions sur ${ }^{14} \mathrm{~N},{ }^{28} \mathrm{Si}$ et ${ }^{29} \mathrm{Si}$. 
Dans le cas de couches très minces, un cas reste sans solution, c'est celui où le même isotope du même élément léger est présent à la fois dans la couche et le substrat, la résolution en profondeur pour cette technique ne pouvant être meilleure que la centaine de nanomètres dans les conditions classiques d'analyse, essentiellement à cause de l'absorbant source de dispersion en énergie. Il faudra alors utiliser le traçage isotopique. C'est un problème classique pour l'oxygène (couche mince d'oxyde sur un oxyde) où l'on utilise couramment l'isotope 18 de l'oxygène comme traceur.

\subsection{Utilisation des résonances fines de réactions nucléaires}

Dans la gamme d'énergie accessible, un certain nombre de réactions présentent un ou des pics fins (largeur $<2 \mathrm{keV}$ ) de section efficace. Les particules émises par cette réaction vont provenir essentiellement de la profondeur où les ions incidents ont atteint, après perte d'énergie lors de leur parcours aller, l'énergie de la résonance.

Ce phénomène est à la base de la technique d'analyse par balayage en énergie. Dans ce cas, toutes les particules (ou les photons $\gamma$ ) issues de la réaction considérée sont comptées en fonction de l'énergie du faisceau incident : on obtient ainsi une courbe d'excitation qui est l'image du profil de concentration de l'isotope de l'élément dans la cible.

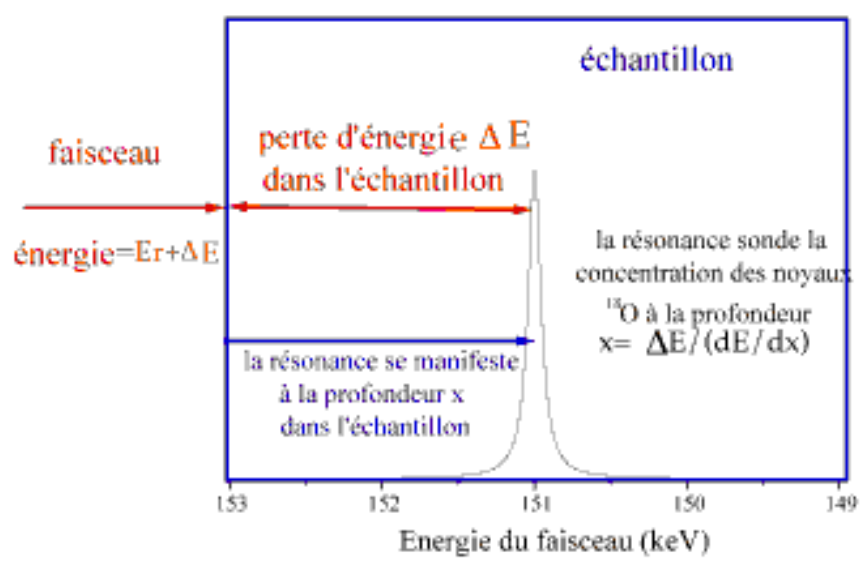

FIG. 18. Principe de la technique de balayage en énergie autour d'une résonance fine de réaction nucléaire.

La résolution en profondeur de cette technique est parmi les meilleures que l'on peut obtenir en microanalyse nucléaire puisque l'on n'a pas besoin d'analyser précisément l'énergie des particules détectées, mais il faut que le faisceau d'analyse ait une faible dispersion en énergie et que la largeur de la résonance soit elle aussi très faible. Par exemple, le profil de concentration d'oxygène 18 dans la silice en utilisant la résonance à $150 \mathrm{keV}$ de la réaction ${ }^{18} \mathrm{O}(\mathrm{p}, \alpha)^{15} \mathrm{~N}$ (de largeur inférieure à $100 \mathrm{eV}$ ) avec un faisceau d'une dispersion du même ordre de grandeur est déterminé avec une précision de environ $1 \mathrm{~nm}$, près de la surface.

Cette très bonne résolution de dégrade avec la profondeur analysée à cause de la dispersion en énergie du faisceau incident lors de sa traversée de la matière. Typiquement, au delà de quelques centaines de nanomètres, la résolution est fortement dégradée. Cette technique est bien adaptée à la mesure de profils de concentration dans des couches minces ou dans la zone tout à fait superficielle des échantillons. 


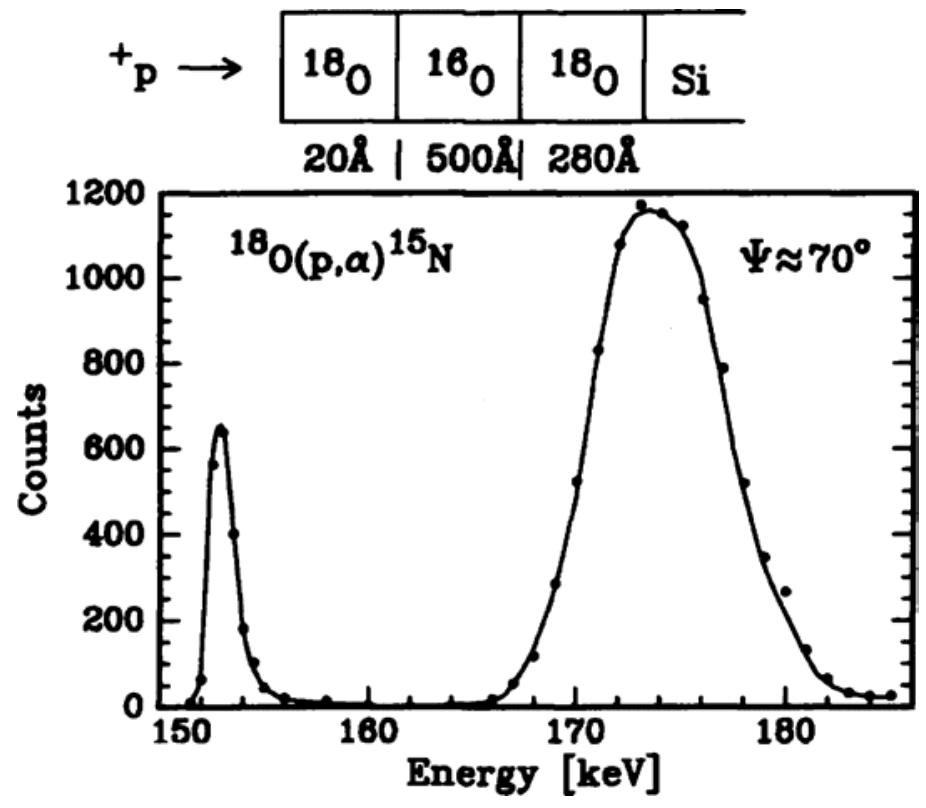

FIG. 19. Exemple de courbe d'excitation obtenue sur un échantillon de silicium oxydé successivement dans l'oxygène 16 puis dans l'oxygène 18 à l'aide de la résonance à $150 \mathrm{keV}$ de la réaction ${ }^{18} \mathrm{O}(\mathrm{p}, \alpha)^{15} \mathrm{~N}$.

\section{ANALYSE PAR SPECTROMÉTRIE DES RAYONS X ÉMIS (PIXE)}

Lors du passage des ions du faisceau incident dans la matière, de nombreuses ionisations des atomes de la cible sont créées. Le retour à l'état fondamental de ceux-ci est accompagné de l'émission de rayons X d'énergie caractéristique de l'élément correspondant. Le spectre des rayons X émis va donc être caractéristique de la composition de l'échantillon. Pour ne pas être gêné par le bruit de fond, on utilise essentiellement les rayons $\mathrm{X}$ émis à la suite d'une ionisation en couche $K$ ou $L$. Les sections efficaces sont ici très grandes et donc la sensibilité est excellente, la détection d'impuretés de l'ordre du ppm est possible.

On peut représenter la section efficace d'ionisation $\sigma$, et donc d'émission de rayons $\mathrm{X}$, par une courbe « universelle » si l'on porte $\frac{\sigma u^{2}}{Z_{1}^{2}}$ en fonction de $\frac{E M_{p}}{u M_{1}}, u$ étant l'énergie de liaison de l'électron, $Z_{1}$ le numéro atomique de la particule, $M_{1}$ sa masse et $E$ son énergie, $M_{P}$ étant la masse du proton.

Le fait d'utiliser des ions et non des électrons a l'avantage de réduire beaucoup le bruit de fond dû au rayonnement de freinage.

Par cette technique, on obtient un comptage global sur l'ensemble du parcours des ions incidents ; la détermination précise d'un profil de concentration est impossible. On peut obtenir une détermination précise de ce contenu global en tenant compte de différents facteurs : la fluorescence $\mathrm{X}$ secondaire, l'absorption des X par la cible elle-même ainsi que par la fenêtre évitant l'arrivée des ions rétrodiffusés sur le détecteur, les variations de section efficace, la perte d'énergie des particules incidentes. Il est souvent utile de se servir pour étalonnage de cibles références bien connues de compositions assez proches de celle de l'échantillon analysé.

À part sa très grande sensibilité, un des intérêts de cette technique où l'on atteint couramment une résolution en énergie de moins de $200 \mathrm{eV}$, est de permettre de différencier des composants lourds les uns des autres quand d'autres techniques, comme la RBS, ne le permettent pas à cause de la faible différence relative de masse (voir l'exemple de la figure 21). 


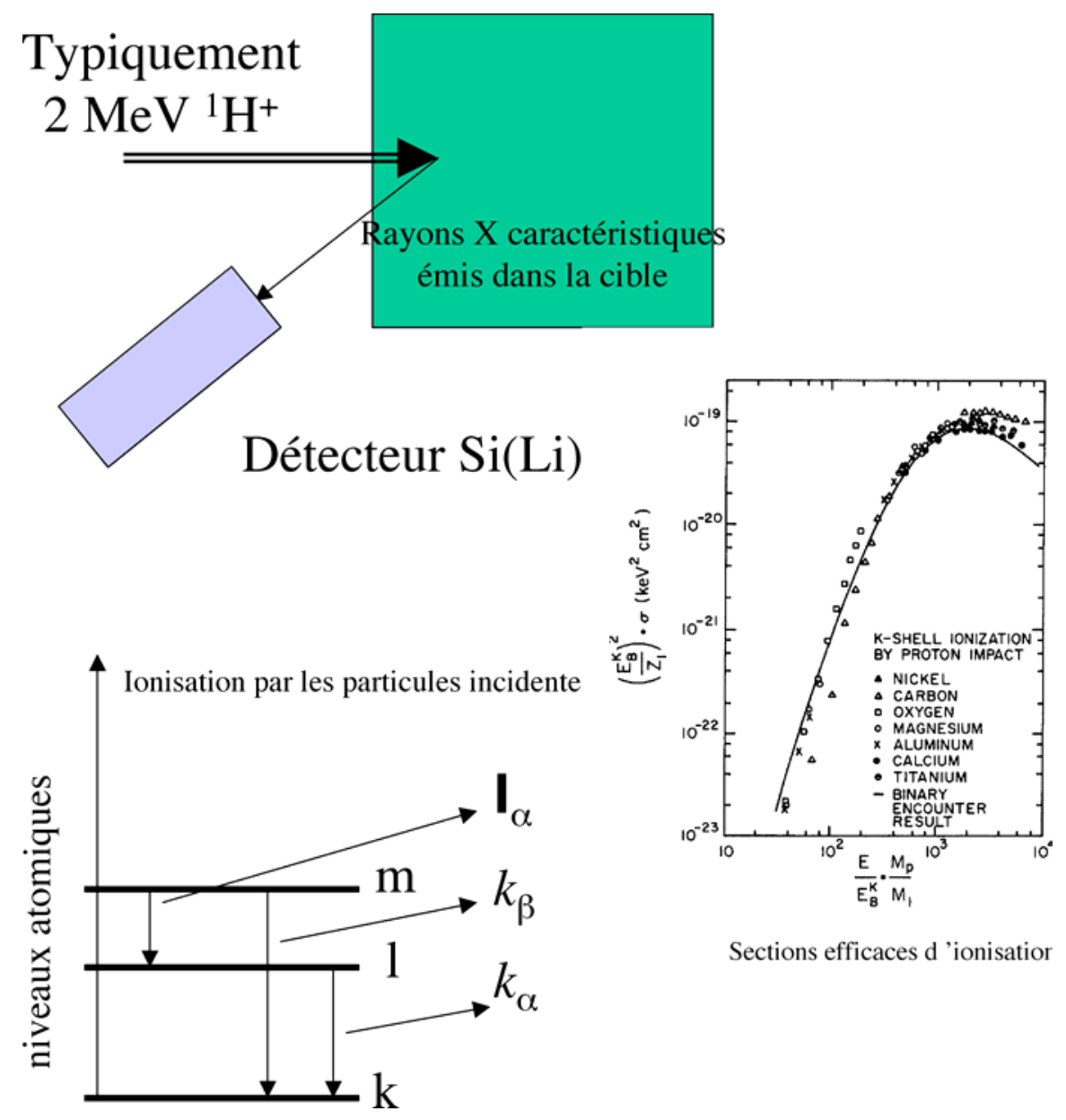

FIG. 20. Principe de la technique PIXE et courbe universelle de section efficace.

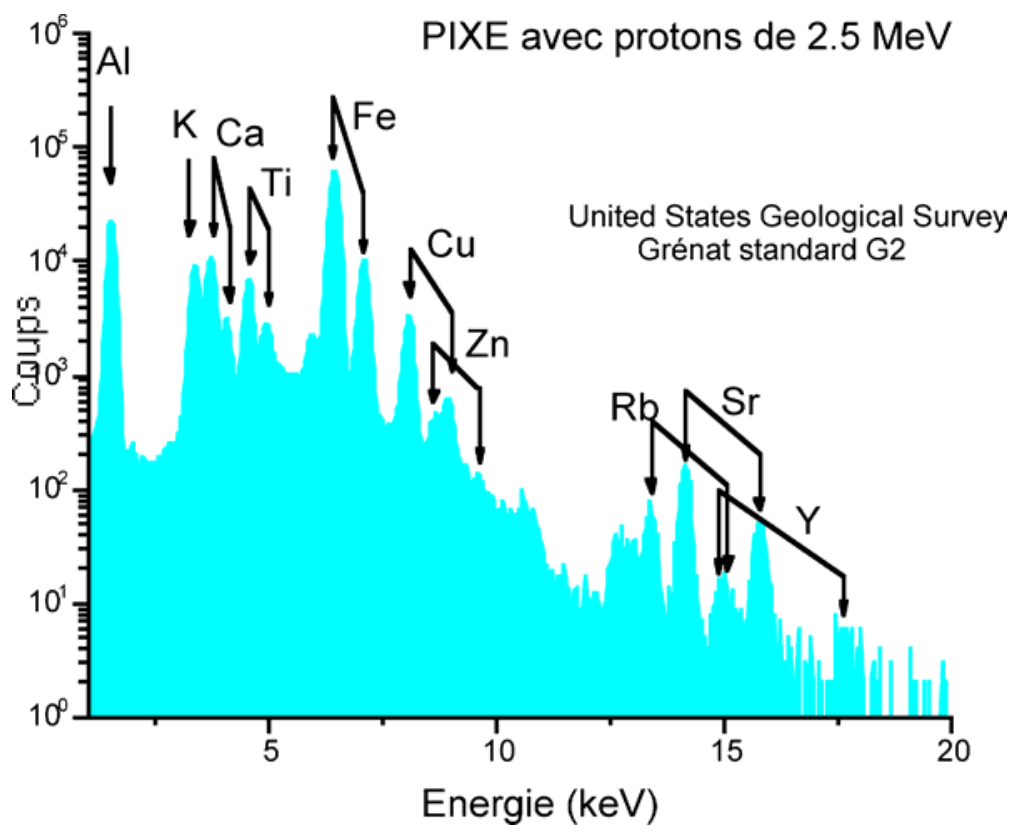

FIG. 21. Exemple de spectre PIXE obtenu sur un composé complexe. On remarque la différenciation entre les éléments $\mathrm{Rb}$, $\mathrm{Sr}$ et $\mathrm{Y}$ de masses et de numéros atomiques très proches. 


\section{QUELQUES COMMENTAIRES SUR CES TECHNIQUES}

Les différentes techniques présentées sont complémentaires les unes avec les autres : éléments lourds, éléments légers, profils de concentration, analyses de traces. Elles ne sont évidemment pas la panacée de la caractérisation des couches minces. En particulier, vu les énergies mises en jeu dans les processus, elles ne sont pas sensibles aux liaisons chimiques qui ne vont intervenir que faiblement ou même pas du tout sur les pouvoirs d'arrêt. Ceci est un avantage pour l'analyse quantitative élémentaire, mais l'information chimique n'est pas présente. La profondeur analysée avec précision est de l'ordre du micron, alors qu'une technique comme le SIMS peut aller plus loin.

Dans la plupart des cas, la dimension latérale du faisceau d'analyse est de l'ordre du $\mathrm{mm}^{2}$. La détermination de la composition des couches sera faite en moyennant sur cette surface.

Il existe des installations où, par focalisation, la taille du faisceau d'analyse peut être réduite à des dimensions de l'ordre de, ou même très inférieures, au micron. Dans ces cas, il est donc possible d'imager élément par élément la zone superficielle de la cible.

\subsection{Problème de la non destructivité}

Ces techniques n'induisent pas, sauf cas particuliers (désorption d'éléments sous le faisceau, qui se produit par exemple dans les polymères), de modification dans la composition de l'échantillon. Le nombre de réactions nucléaires par exemple est extrêmement faible. Typiquement, au maximum un millionième des atomes aura changé de nature dans la trace du faisceau et les diffusions élastiques ne modifient pas la nature des atomes. La répartition spatiale des atomes de la cible ne sera pas modifiée, le nombre de collisions entraînant une mise en mouvement des atomes de la cible étant très faible dans les conditions d'analyse classiques. Une analyse élémentaire par une autre technique sera donc possible sur le même échantillon au même point.

Par contre, les dégâts créés (défauts ponctuels, cassures de liaisons, etc) peuvent modifier les propriétés physiques des échantillons et les rendre inutilisables pour d'autres études (électriques, optiques, magnétiques par exemple). Il peut arriver, dans le cas des faisceaux focalisés, que la grande densité d'énergie déposée par le faisceau d'analyse induise des modifications de profil de concentration, même dans la composition latérale.

\subsection{Interprétation des spectres et dépouillement des résultats}

Pour toutes ces techniques, outre les cas simples où l'on peut simplement comparer les intégrales des pics pour déterminer le contenu d'une couche mince (voir ci-dessus § 3.1.3.1.), on va utiliser des programmes de simulation pour interpréter les spectres obtenus. Schématiquement, ces programmes fonctionnent sur le principe suivant : on suppose une composition de l'échantillon, variable avec la profondeur si nécessaire, on donne les paramètres (nature, énergie et dose des ions, géométrie de l'expérience, nature et épaisseur de l'absorbant devant le détecteur s'il y a lieu). L'échantillon est " découpé » en tranches parallèles à la surface, chaque tranche ayant une composition homogène. Pour chacune des tranches, le programme calcule l'énergie moyenne des ions y parvenant et le nombre de particules émises dans la direction et l'angle solide considérés puis l'énergie perdue lors du parcours avant détection. On obtient ainsi le spectre simulé $N\left(E_{d}\right)$ pour la composition supposée. On fait varier, manuellement ou automatiquement, les compositions des différentes tranches jusqu'à obtenir l'ajustement de la simulation à l'expérience, le spectre simulé étant la somme des contributions au spectre de toutes les tranches de matière (voir la figure 22 à titre d'exemple).

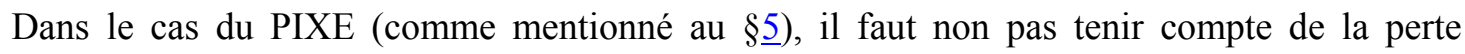
d'énergie à la sortie mais de l'absorption des photons X.

Pour la technique de balayage en énergie autour d'une résonance, l'intégrale des particules émises pour chaque énergie des ions incidents est calculé. Il est indispensable dans ce cas de prendre en compte précisément la dispersion en énergie des ions incidents à la traversée de la matière. 


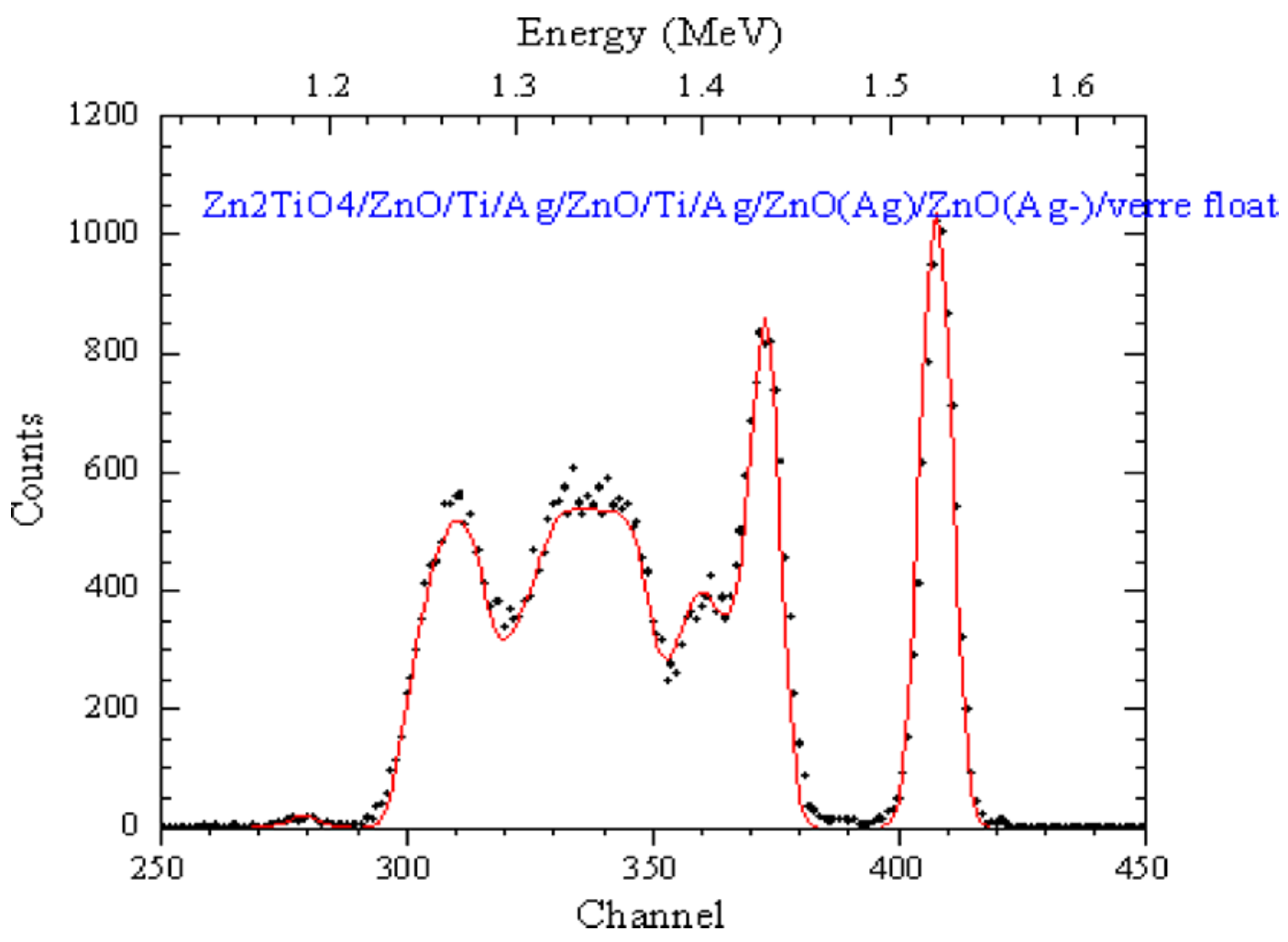

FIG. 22. Exemple de dépouillement d'un spectre RBS obtenu sur un dépôt multi-couches complexe sur verre. En points : expérience, en ligne continue : ajustement. Seule la partie haute énergie du spectre correspondant aux éléments $\mathrm{Ag}, \mathrm{Zn}$, Ti est représentée.

\section{BIBLIOGRAPHIE (quelques ouvrages avec beaucoup de références à l'intérieur)}

L.C. Feldman et J.W. Mayer, Fundamentals of surface and thin films analysis, North-Holland (1986).

W.K. Chu, J.W. Mayer et M.A. Nicolet, Backscattering spectrometry, Academic Press, New York (1978).

L.C. Feldman, J.W. Mayer et S.T. Picraux, Materials analysis by ion channeling, Academic Press, New York (1982).

J.R. Tesmer et M. Nastasi Eds., Handbook of modern ion beam analysis, Material Research Society (1995).

J.W. Mayer et E. Rimini, Ion beam handbook for material analysis, Academic Press, New York (1977).

B. Agius, M. Froment et co-auteurs, Surfaces, interfaces et films minces. Observation et analyse, Dunod (1990).

J.M. Walls Ed., Methods of surface analysis, Cambridge University Press (1989).

J. Tirira, Y. Serruys et P. Trocellier, Forward recoil spectroscopy. Applications to hydrogen determination in solids, Plenum Press (1996). 